\title{
Rheo-Optical Studies on the Deformation Mechanism of Semi- crystalline Polymers XVI. Alpha and Beta Mechanical Dispersions of Spherulitic Polypropylene and Polybutene-1 and the Dynamic Orientation Distribution Function of Crystallites ${ }^{\dagger}$
}

\author{
Ken-ichi FuJITA, ${ }^{* 1}$ Masayoshi DaIo, ${ }^{* 2}$ Ryozo OKUMURA, ${ }^{* 3}$ \\ Shoji SueHIRO, Shunji NomURA, ${ }^{* 4}$ \\ and Hiromichi KAWAI*5 \\ Department of Polymer Chemistry, Faculty of Engineering, \\ Kyoto University, Kyoto 606, Japan
}

(Received February 22, 1982)

\begin{abstract}
The uniaxial deformation mechanism of spherulitic polypropylene and polybutene-1 was investigated in regard to changes in the birefringence and orientation distribution function of crystal grains with the extension ratio of the bulk specimen. This mechanism can be represented by a spherulite deformation model combining the affine orientation of the crystal lamellae with the reorientation of the crystal grains within the orienting lamellae. The latter is caused by three kinds of intra-lamellar shearing mechanisms; i.e., lamellar detwisting mostly in the equatorial zone of the uniaxially deformed spherulites, and lamellar tilting and micronecking mostly in the polar zone of the spherulites. In contrast to spherulitic high-density polyethylene in which the lamellar detwisting and tilting mechanisms are dominant, the lamellar tilting and micronecking occur dominantly in spherulitic polypropylene and polybutene-1. The investigation was extended to dynamic measurements and good correspondence of the mechanical and optical dispersions designated as $\alpha$ and $\beta$ dispersions was found, in contrast to the case of spherulitic highdensity polyethylene for which an additional $\alpha_{2}$ mechanical dispersion having no correspondence to the optical dispersion was observed. The $\beta$ dispersion became clearer the $\alpha$ dispersion did less so as the specimen changed from polyethylene to polypropylene and polybutene-1. The $\beta$ dispersion was attributed to the dynamic orientation dispersion of the crystal lamellae constituting the spherulitic crystalline texture for any poly-alpha-olefin specimen, i.e., inter-lamellar crystal-grain-boundary phenomena possibly associated with orientational and/or distorsional dispersions of noncrystalline material between the lamellae. The $\alpha$ dispersion was ascribed to the dynamic reorientation dispersion of the crystal grains within the orienting lamellae, which occurs with a preferential orientation of the $c$-axis caused by dynamic lamellar detwisting and tilting in the case of polyethylene and only by dynamic lamellar tilting for polypropylene and polybutene- 1 , i.e., intralamellar crystal-grain-boundary phenomena associated with the inter-crystal friction at the boundaries.
\end{abstract}

+ Presented in part at the IUPAC 28th Macromolecular Symposium, University of Massachusetts, Amherst, Mass., July 12-16, 1982 and in part at the ACS Award Symposium for Prof. R. S. Stein, ACS Seattle Meeting, March $20-25,1983$.

*1 Present address: Department of Polymer Engineering, Nagoya Institute of Technology, Gokiso, Showa-ku, Nagoya 466, Japan.

*2 Present address: Materials Research Department, Yokohama Rubber Co., 2-1, Oiwake, Hiratsuka 254, Japan.

*3 Present address: Polymer Research Laboratory, Idemitsu Petrochemical Industries, 1-1, Anesaki-Kaigan, Ichihara, Chiba 299-01, Japan.

*4 Present address: Department of Textile Engineering, Kyoto University of Industrial Arts and Fiber Technology, Matsugasaki, Sakyo-ku, Kyoto 606, Japan.

*5 To whom correspondence should be addressed. 


\begin{abstract}
KEY WORDS Rheo-Optical Properties / Polypropylene / Polybutene-1 / Spherulite Deformation Mechanism / Alpha and Beta Mechanical Dispersions / Dynamic Orientation Distribution Function of Crystallites /
\end{abstract}

In the previous papers ${ }^{1,2}$ of this series dealing with the deformation mechanism of semicrystalline polymers, the uniaxial deformation mechanism of spherulitic and row-nucleated high-density polyethylene was investigated, observing the changes in the orientation distribution function of crystallites (crystal grains), $w(\xi, 0, \eta)$, with the extension ratio of the bulk specimens. Thus, the dependence of $w(\xi, 0, \eta)$ on the extension ratio was compared with that calculated from a spherulite deformation model which combines the affine crystal lamellar orientation with the reorientation of the crystal grains within the orienting lamellae. The crystal reorientation mechanism is discussed in terms of two types of rotation of the crystal grains about their own $a$ - and $b$-axes, respectively, both so as to orient their $c$-axes toward the stretching direction. The rotations of the crystal grains about the $a$ - and $b$-axes must be associated with two types of intralamellar shearing, lamellar tilting and detwisting, and must be accentuated in the polar and equatorial zones of the uniaxially deformed spherulites. ${ }^{1}$

These postulations on the uniaxial deformation mechanism of the spherulitic crystalline texture of polyethylene, especially the lamellar detwisting mechanism in the equatorial zone of the spherulites, was confirmed experimentally by investigating the uniaxial deformation behavior of a rownucleated polyethylene in terms of the change in $w(\xi, 0, \eta)$ with the extension ratio of the specimen in the direction of the row. ${ }^{2}$ Since the row-nucleated specimen has a particular crystalline texture consisting of cylindrites in which the radially grown crystal lamellae are stacked with their axes highly oriented perpendicular to the direction of the row, the uniaxial deformation mechanism of the row-nucleated specimen must represent the mechanism expected in the equatorial zone of the uniaxially deformed spherulites.

On the basis of the uniaxial deformation mechanism of polyethylene spherulites mentioned above, the dynamic uniaxial deformation mechanism of spherulitic high-density polyethylene was investigated in terms of the dynamic change in the orientation distribution function of the crystal grains,
$\Delta w^{*}(\xi, 0, \eta)$, in order to assign the $\alpha$ and $\beta$ mechanical dispersions of polyethylene. ${ }^{3}$ The temperature and frequency dependence of the dynamic change in the orientation distribution function was analyzed further, using the dynamic response of the parameters of the spherulite deformation model, and the following assignment was deduced. The $\alpha$ mechanical dispersion must be assigned to the dynamic orientation dispersion of the crystal grains within the crystal lamellae. This orientation is caused by two types of dynamic rotation of the grains about their crystal $b$ - and $a$-axes associated, respectively, with dynamic lamellar detwisting and dynamic lamellar tilting, i.e., both intra-lamellar crystal-grain-boundary phenomena. The $\beta$ mechanical dispersion must be assigned to the dynamic orientation dispersion of the crystal lamellae themselves behaving as rigid bodies. This dispersion is not associated with any dynamic reorientation of the crystal grains, but with the dynamic orientation dispersion of noncrystalline material between the lamellae, i.e., inter-lamellar crystalgrain-boundary phenomena.

We were interested in extending such static and dynamic studies on the deformation mechanism of the spherulitic crystalline texture to isotactic polypropylene and polybutene- 1 in order to characterize the deformation mechanism in these particular polymers and farther to obtain basic information on the dynamic mechanical dispersions of a series of poly-alpha-olefins. In this paper, the uniaxial deformation mechanism of spherulitic polypropylene and polybutene- 1 is investigated first in terms of the change in the orientation distribution function of the crystal grains, $w(\xi, 0, \eta)$ with the extension ratio of the bulk specimens. The dynamic rheooptical properties, of well-annealed spherulitic polypropylene and polybutene-1 were investigated using dynamic birefringence ${ }^{4}$ and dynamic $\mathrm{X}$-ray diffraction ${ }^{5}$ techniques over sufficiently wide ranges of temperature and frequency. On the basis of the data obtained the $\alpha$ and $\beta$ mechanical dispersions of these particular specimens were assigned and compared with those of spherulitic high-density polyethylene. 
The Mechanical Dispersion and the Dynamic Crystal Orientation of Spherulitic PP and PB-1

\section{TEST SPECIMENS AND EXPERIMENTAL PROCEDURES}

\section{Preparation of Test Specimens}

Commercial pellets of isotactic polypropylene (Polypropylene B-200, Mitsui Petrochemical Ind., Ltd.) and isotactic polybutene-1 (Polybutene M801N, Mitsui Petrochemical Ind., Ltd.) were placed in a spacer sandwiched between polished stainless-steel plates, and molten in a laboratory hot-press for several minutes under a pressure of $200 \mathrm{~kg} \mathrm{~cm}^{-2}$. The stainless steel plates containing the melt were removed from the press and immediately plunged into water to obtain a quenched specimen in sheet form. This quenched specimen was annealed at a particular elevated temperature in a vacuum oven and gradually cooled down to ambient temperarture. The thickness of the respective specimens was adjusted by a spacer to about $500 \mu \mathrm{m}$ for X-ray diffraction measurements and to $150 \mu \mathrm{m}$ for birefringence measurements. The preparation conditions and characterization of the specimens are listed in Table I, along with the specimen codes for the respective polymers.

Figure 1(a) shows the $\mathrm{X}$-ray diffraction intensity distribution as a function of the Bragg angle $2 \theta_{B}$ for the polypropylene specimen it-PP B-200(A) prepared under the conditions listed in Table I. Figures 1(b) and 1(c) show the corresponding distributions for other specimens prepared from the same polymer under different conditions; i.e., (b) quenched from the melt at $230^{\circ} \mathrm{C}$ into dry-ice methanol of $-75^{\circ} \mathrm{C}$ and annealed at $130^{\circ} \mathrm{C}$ for $4 \mathrm{~h}$, and (c) quenched from the melt at $230^{\circ} \mathrm{C}$ into dry-ice

Table I. Preparation and characterization of test specimens

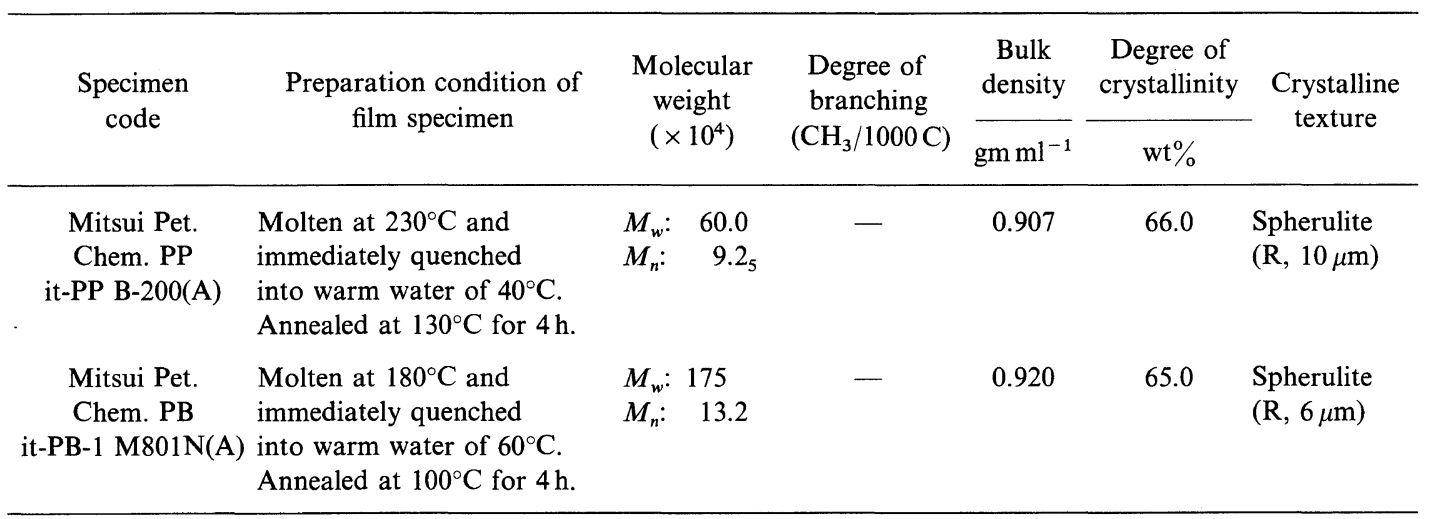

a Determined by water/methanol density gradient tube at $25.0^{\circ} \mathrm{C}$.

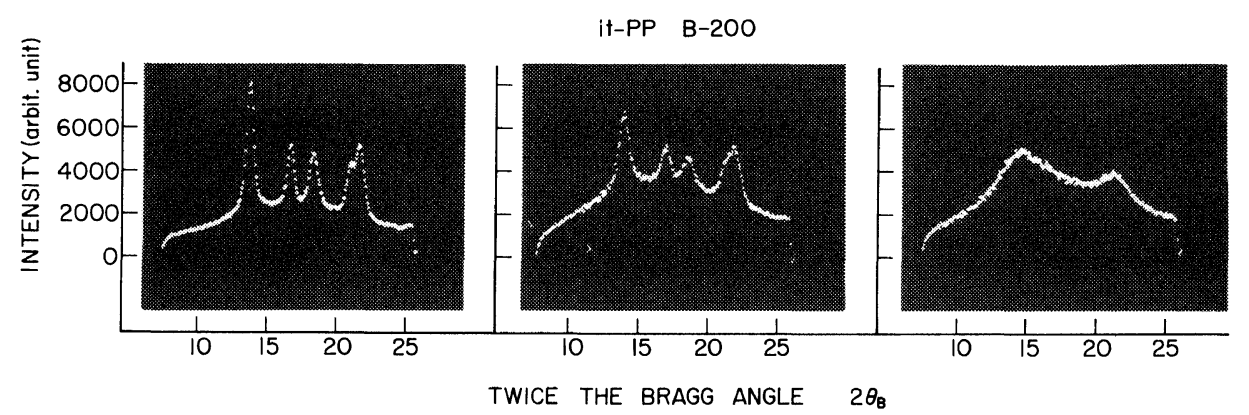

(a)

(b)

(c)

Figure 1. X-Ray diffraction intensity distributions of polypropylene specimens prepared under different conditions: (a) quenched into warm water of $40^{\circ} \mathrm{C}$ and annealed at $130^{\circ} \mathrm{C}$ for $4 \mathrm{~h}$; (b) quenched into dry-ice methanol bath of $-75^{\circ} \mathrm{C}$ and annealed at $130^{\circ} \mathrm{C}$ for $4 \mathrm{~h}$; and (c) quenched into dry-ice methanol bath. 


\section{K. FuJita et al.}

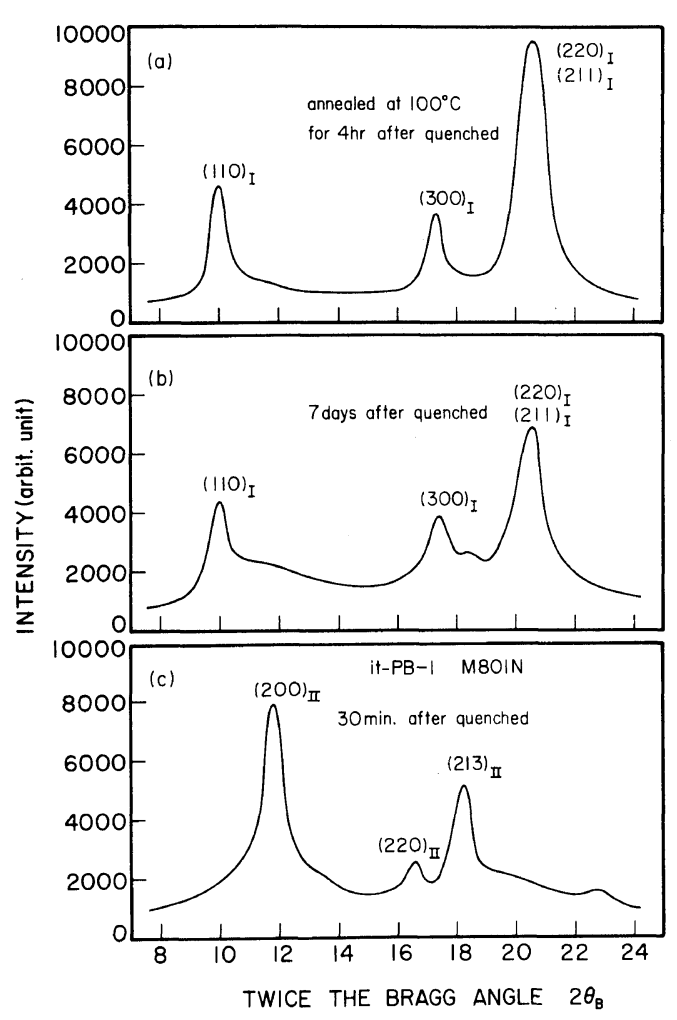

Figure 2. X-Ray diffraction intensity distribution of polybutene-1 specimens prepared under different conditions: (a) quenched into warm water of $60^{\circ} \mathrm{C}$ and annealed at $100^{\circ} \mathrm{C}$ for $4 \mathrm{~h}$; (b) quenched into warm water of $60^{\circ} \mathrm{C}$ and stayed at room temperature for a week; and (c) quenched into warm water of $60^{\circ} \mathrm{C}$ and stayed at room temperature for $30 \mathrm{~min}$.

methanol without any further annealing. As can be seen from the figures, the specimen it-PP B-200(A) has a relatively high degree of crystallinity with an almost completely monoclinic crystal system, while the quenched specimen (c) is of a relatively low degree of crystallinity with the so-called smectic crystal system, and the quenched and annealed specimen (b) is between (a) and (c).

Figure 2(a) shows the X-ray diffraction intensity distribution as a function of $2 \theta_{\mathbf{B}}$ for the polybutene1 specimen it-PB-1 M801N(A) was prepared under the conditions listed in Table I. Figures 2(b) and 2(c) show the data for other specimens prepared from the same polymer under different conditions; i.e., (b) quenched from the melt at $180^{\circ} \mathrm{C}$ into water of $60^{\circ} \mathrm{C}$ and left at room temperature for one week, and (c) quenched from the melt at $180^{\circ} \mathrm{C}$ into water of $60^{\circ} \mathrm{C}$ and left at room temperature for only $30 \mathrm{~min}$. As can be seen from these figures, the specimen it-PB-1 M801N(A) has a relatively high degree of crystallinity with an almost hexagonal crystal system, while the quenched specimen (c) is of a tetragonal crystal system, and specimen (b) is of a rather hexagonal crystal system transformed from the tetragonal system of the specimen (c).

By polarized light-scattering the well-annealed specimens, it-PP B-200(A) and it-PB-1 M801N(A), were found to have well-developed spherulitic crystalline textures whose $H_{\mathrm{v}}$ and $V_{\mathrm{v}}$ scattering patterns are shown in Figure 3. From the $H_{\mathrm{v}}$ patterns the average radius of the spherulites was estimated to be about 10 and $6 \mu \mathrm{m}$ for the respective specimens, ${ }^{6,7}$ and from the $V_{\mathrm{v}}$ scattering patterns, both specimens were found to have the so-called negative spherulites within which the refractive index in the radial direction is smaller than that in the tangential direction. ${ }^{8}$ The densities of the respective specimens were determined to be 0.908 and $0.920 \mathrm{~g} \mathrm{~cm}^{-3}$ by a methanol-water density gradient column at $25.0^{\circ} \mathrm{C}$. The degree of crystallinity was estimated from the density values to be 66.0 and $65.0 \mathrm{wt} \%$, assuming the densities of the crystalline and noncrystalline regions to be 0.936 and $0.855 \mathrm{~g} \mathrm{~cm}^{-3}$ and 0.950 and $0.870 \mathrm{~g} \mathrm{~cm}^{-3}$ for the respective polymers. ${ }^{9}$

\section{Experimental Procedures}

The well-annealed sheet specimens, it-PP B200(A) and it-PB-1 M801N(A), of $150 \mu \mathrm{m}$ in thickness were cut into a ribbon shape of $8 \mathrm{~cm}$ long and $1 \mathrm{~cm}$ wide for static birefringence measurements as a function of extension ratio and for dynamic mechanical and birefringence measurements as a function of frequency at a given extension ratio. The well-annealed sheet specimens of $500 \mu \mathrm{m}$ in thickness were also cut into ribbons $10 \mathrm{~cm}$ long and $1.5 \mathrm{~cm}$ wide for static X-ray diffraction measurements as a function of extension ratio and for dynamic X-ray diffraction measurements as a function of frequency at a given extension ratio.

For the static birefringence and X-ray diffraction measurements, the ribbon shape specimens were stretched uniaxially up to given extension ratios at fixed temperatures and extension rates as low as 1 to $1.5 \% \mathrm{~min}^{-1}$. The maximum extension ratios for the respective specimens were the limits of uniform deformation beyond which the specimens exhibited macroscopic necking. The stretched specimens thus 
The Mechanical Dispersion and the Dynamic Crystal Orientation of Spherulitic PP and PB-1

$$
\text { it-PP B-200(A) }
$$

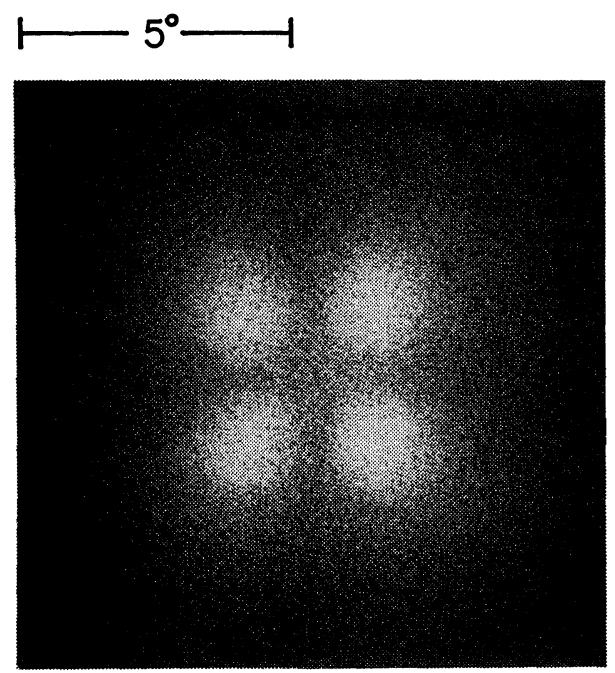

$H_{V}$

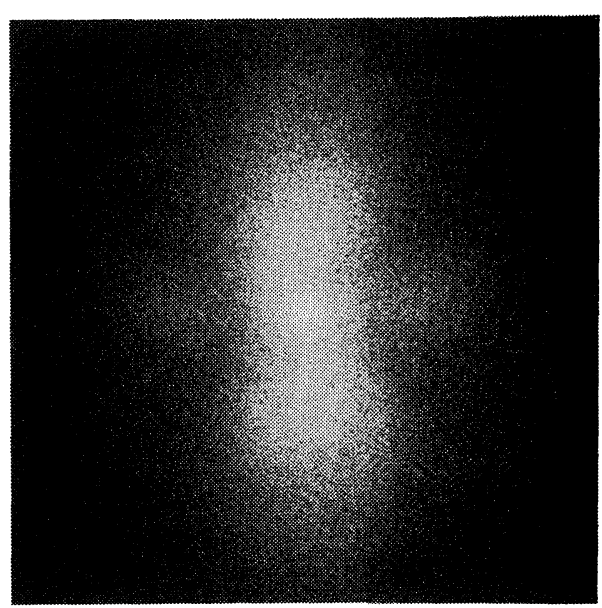

$V_{V}$

\section{it-PB-I M8OIN(A)}

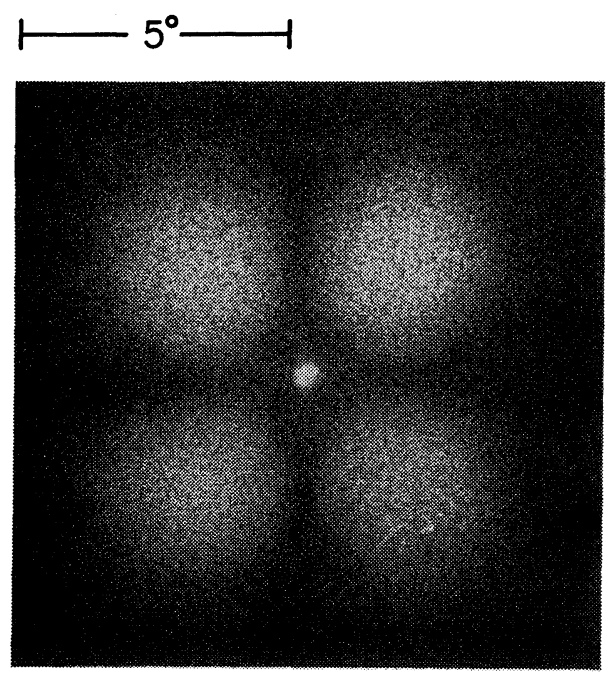

$\mathrm{H}_{\mathrm{V}}$

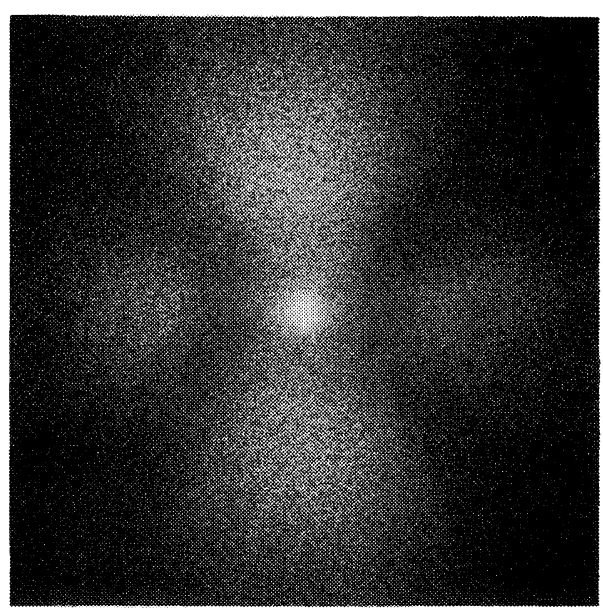

$\mathrm{V}_{\mathrm{V}}$

Figure 3. Polarized light scattering patterns from well-annealed specimens of polypropylene and polybutene-1, it-PP B-200(A) and it-PB-1 M801N(A), under $H_{\mathrm{v}}$ and $V_{\mathrm{v}}$ polarization conditions. 


\section{K. FujITA et al.}

obtained were clamped between two metalic flanges and kept at room temperature for about one week prior to static birefringence and X-ray diffraction measurements. X-Ray diffraction measurements were carried out using the $\mathrm{Cu} K \alpha$ line of X-ray beam generated from a rotating anode type generator (RU-200Z Rigaku Denki Co., Tokyo) with a power of $40 \mathrm{kV}$ and $200 \mathrm{~mA}$. Measurement of the X-ray diffraction intensity distribution was performed for 7 and 6 different crystal planes for it-PP B-200(A) and it-PB-1 M801N(A) (see II) using an ordinary horizontal scanning type goniometer operating at a fixed time step scan of 0.1 or $1^{\circ}$ interval over ranges of $2 \theta_{\mathrm{B}}$ from 10 to $32^{\circ}$ and 6 to $37^{\circ}$ for the respective specimens, with the surface normal to the ribbon shape specimen tilting by an angle $\theta_{\mathbf{B}}$ with respect to the incident $\mathrm{X}$-ray beam. The horizontal scanning was performed at various azimuthal angles $\psi$ by rotating the specimen in a stepwise fashion about the normal of the surface at $5^{\circ}$ intervals. In this way, the orientation distribution of the reciprocal lattice vector for the $j$-th crystal planes within the plane parallel to the specimen surface was obtained. The orientation distribution obtained was sufficient for a cylindrically symmetric system of a specimen uniaxially stretched in a particular direction. Practical procedures for determining the uniaxial orientation distribution function of reciprocal lattice vector for the $j$-th crystal planes, $q_{j}\left(\zeta_{j}, 0\right)$, from the measured X-ray diffraction intensity distribution $I\left(2 \theta_{\mathrm{B}}, \psi\right)$ are described elsewhere. ${ }^{1,10}$ These procedures are based on the assumption that the diffraction intensity distribution from the $j$-th crystal plane, $I_{j}\left(2 \theta_{\mathrm{B}}, \psi_{0}\right)$, follows a Gaussian type function.

For dynamic mechanical and birefringence measurements, the ribbon shape specimen of $150 \mu \mathrm{m}$ in thickness was mounted on a tensile dynamic deformation apparatus at an initial gauge length of $6 \mathrm{~cm}$ and subjected to a static tensile strain of $3.3 \%$ to prevent the specimen from buckling when a dynamic tensile strain of $0.25 \%$ was applied. A preliminary vibration was applied at $4.3 \mathrm{~Hz}$ for one hour to attain a vibrational steady state and to provide the particular mechanical conditions necessary for the dynamic measurements. The simultaneous measurements of dynamic mechanical and dynamic birefringence behavior were conducted by means of a $\pi$-sector technique 4 over a frequency range from 0.008 to $4.3 \mathrm{~Hz}$ at various temperatures from -40 to $100^{\circ} \mathrm{C}$.
For dynamic X-ray diffraction measurements, the ribbon shape specimen of $500 \mu \mathrm{m}$ in thickness was mounted in another tensile dynamic deformation apparatus at a gauge length of $4 \mathrm{~cm}$. It was cut into thin strips each about $3 \mathrm{~mm}$ wide along the gauge length to avoid biaxial deformation of the specimen and a static tensile strain of $5 \%$ was superposed on a dynamic tensile strain of $0.46 \%$. A preliminary vibration was also applied for one hour at $0.2 \mathrm{~Hz}$. The measurements were carried out using a multichannel narrow-sector technique ${ }^{5}$ at a frequency of $0.2 \mathrm{~Hz}$ by varying the temperature from -20 to $100^{\circ} \mathrm{C}$ at $10^{\circ} \mathrm{C}$ intervals. The dynamic measurements were performed by using a dynamic diffractometer $^{11}$ in a fixed time step-scanning manner over ranges of $2 \theta_{\mathrm{B}}$ from 12 to $24^{\circ}$ and from 8 to $19^{\circ}$ for the it-PP B-200(A) and it-PB-1 M801N(A) specimens, respectively, both at $0.2^{\circ}$ intervals and over a range of $\psi$ from 0 to $90^{\circ}$ at $22.5^{\circ}$ intervals. This was done so as to investigate the dynamic change in the uniaxial orientation distribution function $\Delta q_{j}^{*}\left(\zeta_{j}, 0\right)$ of the (110), (040), (130), and (111) crystal planes for the it-PP B-200(A), and the (110) and (300) crystal planes for the it-PB-1 M801(A), and also in that of the crystal grains, $\Delta w^{*}(\xi, 0, \eta)$. Detailed procedures for evaluating $\Delta q_{j}^{*}\left(\zeta_{j}, 0\right)$ and $\Delta w^{*}(\xi, 0, \eta)$ are fully discussed elsewhere. ${ }^{3}$

\section{DEFINITION OF THE COMPLEX DYNAMIC ORIENTATION DISTRIBUTION FUNCTION OF CRYSTALLITES AND ITS INVESTI- GATION BY DYNAMIC X-RAY DIFFRACTION}

As depicted in Figure 4(a), the orientation of the Cartesian coordinate system $0-u_{1} u_{2} u_{3}$ fixed within a crystallite (or crystal grain), relative to another Cartesian coordinate system $0-x_{1} x_{2} x_{3}$ fixed within a bulk specimen can be specified in terms of three Euler angles $\phi, \theta$, and $\eta$. The coordinate system 0 $u_{1} u_{2} u_{3}$ must be fixed within the crystal grain to make the system as highly symmetric as possible. Thus, for polypropylene (monoclinic) crystal, the $u_{2}$ and $u_{3}$ axes are taken parallel to the crystal $b$ - and $c^{-}$ axes, respectively, and hence, the $u_{1}$ axis are parallel to the $a^{*}$-axis, not the $a$-axis. For polybutene- 1 (hexagonal) crystal, the $u_{1}$ and $u_{3}$ axes are taken parallel to the crystal $a^{*}$-axis [reciprocal lattice vector of the (300) crystal palne] and the crystal $c$ - 


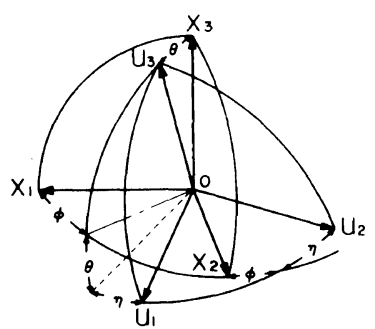

(a)

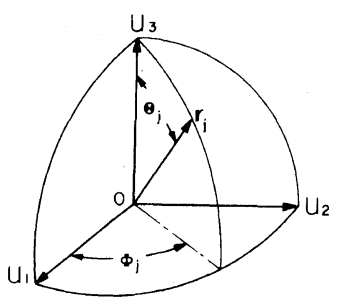

(b)

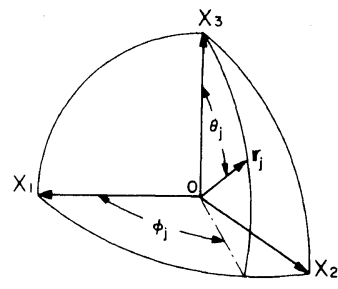

(c)

Figure 4. (a) Three Euler angles, $\phi, \theta$, and $\eta$, specifying the orientation of a Cartesian coordinate system $0-u_{1} u_{2} u_{3}$, fixed within a crystallite, with respect to another Cartesian coordinate system, fixed within the bulk specimen; (b) polar and azimuthal angles, $\Theta_{j}$ and $\Theta_{j}$, specifying the orientation of the reciprocal lattice vector of the $j$-th crystal plane, $r_{j}$ with respect to the Cartesian coordinate system $0-u_{1} u_{2} u_{3}$; and (c) polar and azimuthal angles, $\theta_{j}$ and $\phi_{j}$, specifying the orientation of the $r_{j}$ vector with respect to the Cartesian coordinate system $0-x_{1} x_{2} x_{3}$.

axis, respectively.

The coordinate system $0-x_{1} x_{2} x_{3}$ must be fixed within the bulk specimen so as to make the orientation distribution of the crystal grains as highly symmetric as possible with respect to the coordinate axes. Thus, the axes $x_{3}, x_{2}$, and $x_{1}$ must be taken parallel to the machine, transverse and thickness directions, respectively, for sheet-like specimen stretched orthogonal-biaxially or uniaxially. These choices of coordinate axes are necessary to simplify the mathematical description of the orientation of crystal grains within the bulk specimen. Figures 4(b) and 4(c) specify the orientation of a given vector $\boldsymbol{r}_{j}$ (reciprocal lattice vector of the $j$-th crystal plane) within the crystal grains and the bulk specimen, respectively, in terms of the pairs of polar and azimuthal angles, $\Theta_{j}, \Phi_{j}$ and $\theta_{j}, \phi_{j}$.

The orientation distribution functions of the crystal grains and the $\boldsymbol{r}_{j}$ vectors within the bulk specimen, $w(\theta, \phi, \eta)$ and $q_{j}\left(\theta_{j}, \phi_{j}\right)$, can be defined under the following normalization conditions:

$$
\begin{aligned}
& \int_{\eta=0}^{2 \pi} \int_{\phi=0}^{2 \pi} \int_{\xi=-1}^{1} w(\xi, \phi, \eta) \mathrm{d} \xi \mathrm{d} \phi \mathrm{d} \eta=1 \\
& \int_{\phi_{j}=0}^{2 \pi} \int_{\zeta_{j}=-1}^{1} q_{j}\left(\zeta_{j}, \phi_{j}\right) \mathrm{d} \zeta_{j} \mathrm{~d} \phi_{j}=1
\end{aligned}
$$

where $\xi=\cos \theta$ and $\zeta_{j}=\cos \theta_{j}$.

The orientation distribution functions, $w(\xi, \phi, \eta)$ and $q_{j}\left(\zeta_{j}, \phi_{j}\right)$, can be examined by the well-known mathematical procedure which involves expanding the functions in an infinite series of spherical harmonics, as discussed by Roe and Krigbaum. ${ }^{12-14}$ The cylindrical symmetry (uniaxial symmetry) of the orientation distribution of the $r_{j}$ vectors with respect to the stretching direction ( $x_{3}$ axis) makes the distributions of $\phi$ and $\phi_{j}$ random, which will be denoted as $w(\xi, \phi=0, \eta)$ and $q_{j}\left(\zeta_{j}, \phi_{j}=0\right)$ hereafter. For particular combinations of the cylindrical symmetry of $q_{j}\left(\zeta_{j}, \phi_{j}\right)$ about the $x_{3}$ axis with the monoclinic and hexagonal symmetries of the Cartesian coordinate system $0-u_{1} u_{2} u_{3}$, for uniaxially stretched specimens of polypropylene and polybutene- 1 , the orientation distribution functions can be expanded in an infinite series of spherical harmonics as follows:

$$
\begin{gathered}
w(\xi, 0, \eta)=\sum_{l=0}^{\infty} A_{l 00} \Pi_{l}(\xi)+2 \sum_{l=2}^{\infty} \sum_{n=1}^{l} A_{l 0 n} \Pi_{l}{ }^{n}(\xi) \cos (n \eta) \\
q_{j}\left(\zeta_{j}, 0\right)=\sum_{l=0}^{\infty} \alpha_{l 0}^{j} \Pi_{l}\left(\zeta_{j}\right)
\end{gathered}
$$

where $A_{l m n}$ and $\alpha_{l m}^{j}$ are the real components of the coefficients in each series, $\Pi_{l}{ }^{n}(x)$ is a normalized Legendre polynomial, and $l, m$, and $n$ are integers indicating the order of the expansion. Further, $l$ is an even number and $m$ is zero, while $n$ is an integer and a multiple of six for the monoclinic and hexagonal symmetries of the Cartesian coordinate system, respectively.

The coefficients, $A_{l 0 n}$ and $\alpha_{l 0}^{j}$ are given by

$$
\begin{gathered}
A_{l 0 n}=\frac{1}{2 \pi} \int_{0}^{2 \pi} \int_{-1}^{1} w(\xi, 0, \eta) \Pi_{l}{ }^{n}(\xi) \cos (n \eta) \mathrm{d} \xi \mathrm{d} \eta \\
\alpha_{l 0}^{j}=\int_{-1}^{1} q_{j}\left(\zeta_{j}, 0\right) \Pi_{l}\left(\zeta_{j}\right) \mathrm{d} \zeta_{j}
\end{gathered}
$$

As can be seen from eq 5 and 6 , the coefficients are the averages of the distribution functions with respect to the spherical harmonics of the $l 0 n$-th and 
10-th orders, respectively. In other words, the coefficients are explicitly the $l 0 n$-th and $l 0$-th moments of the orientation distribution functions.

By applying a generalized Legendre addition theorem to the relation between the sets of polar and azimuthal angles, $\Theta_{j}, \Phi_{j}$ and $\theta_{j}, \phi_{j}$ through the Euler angles, $\phi, \theta$, and $\eta$, the following relation can be obtained:

$$
\begin{aligned}
\alpha_{l 0}^{j}= & 2 \pi\left(\frac{2}{2 l+1}\right)^{1 / 2}\left[A_{l 00} \Pi_{l}\left(\cos \Theta_{j}\right)\right. \\
& \left.+2 \sum_{n=1}^{l} A_{l 0 n} \Pi_{l}{ }^{n}\left(\cos \Theta_{j}\right) \cos \left(n \Phi_{j}\right)\right]
\end{aligned}
$$

Equation 7 is one of the most significant results from the above mathematical development, interrelating not only the two coefficients, $\alpha_{l 0}^{j}$ and $A_{l 0 n}$, but also the coefficients $\alpha_{10}^{j}$ with different $j$, as discussed by Sack ${ }^{15}$ and Wilchinsky, ${ }^{16}$ and allowing the orientation distribution function $w(\xi, 0, \eta)$ to be determined from a measurable function $q_{j}\left(\zeta_{j}, 0\right)$ and further from $\alpha_{l 0}^{j}$ with an approximation depending upon the number of $j$.

The uniaxial orientation distribution function, $q_{j}\left(\zeta_{j}, 0\right)$, of the $j$-th crystal plane can be obtained from the X-ray diffraction intensity distribution, $I_{j}\left(\theta_{j}, \phi_{j}=90^{\circ}\right)$, at the Bragg angle of the $j$-th plane, $2 \theta_{\mathrm{B}, j}$, as follows:

$$
\begin{aligned}
q_{j}\left(\zeta_{j}, 0\right)= & I_{j}\left(\theta_{j}, \phi_{j}=90^{\circ}\right) / 4 \pi \\
& \times \int_{0}^{\pi / 2} I_{j}\left(\theta_{j}, \phi_{j}=90^{\circ}\right) \sin \theta_{j} \mathrm{~d} \theta_{j}
\end{aligned}
$$

Once the $q_{j}\left(\zeta_{j}, 0\right)$ is determined, $\alpha_{l 0}^{j}$ can be calculated from eq 6 for any even $l$. Using $\alpha_{10}^{j}$ thus determined and $\Theta_{j}$ and $\Phi_{j}$, which are listed in Table II for the $j$-th crystal plane, the coefficient $A_{l 0 n}$ can be calculated by solving a set of simultaneous equations in eq 7 , varying $j$ up to at least $(l+1)$ and $(l / 6+1)$ for the monoclinic and hexagonal crystal systems, respectively. Further, the orientation distribution function of the crystal grains, $w(\xi, 0, \eta)$, can be determined from eq 3 with the approxi-
Table II. Bragg angle $2 \theta_{\mathrm{B}}$ and polar and azimuthal angles $\Theta_{j}$ and $\Phi_{j}$ in regard to the orientation of the reciprocal lattice vector of the $j$-th crystal plane within the crystal lattice

Polypropylene

\begin{tabular}{ccrr}
\hline$(h k l)$ & $2 \theta_{\mathrm{B}}$ & $\Theta_{j}$ & $\Phi_{j}$ \\
\hline$(110)$ & 14.2 & 90.00 & 17.39 \\
$(040)$ & 16.9 & 90.00 & 90.00 \\
$(130)$ & 18.7 & 90.00 & 42.84 \\
$(111)$ & 21.2 & 49.75 & 15.06 \\
$(131)$ & 24.6 & 56.16 & 38.59 \\
$(200)$ & 27.2 & 90.00 & 0.00 \\
$(11 \overline{2})$ & 29.3 & 159.82 & 24.95 \\
\hline
\end{tabular}

Polybutene-1

\begin{tabular}{rcrr}
\hline$(110)$ & 9.98 & 90.00 & 30.00 \\
$(300)$ & 17.3 & 90.00 & 0.00 \\
$(31 \overline{1})$ & 25.0 & 123.19 & 13.89 \\
$(102)$ & 28.0 & 168.03 & 0.00 \\
$(21 \overline{2})$ & 31.5 & 150.71 & 19.11 \\
$(511)$ & 35.4 & 67.04 & 38.95 \\
\hline
\end{tabular}

mation of a finite series up to orders $l=(j-1)$ and $6(j-1)$ for the respective systems.

When a dynamic tensile excitation with sufficiently small amplitude $\Delta \lambda$ is applied on a bulk specimen under a static strain of $\lambda^{0}$, i.e., when the strain $\lambda(t)$ is given by

$$
\lambda(t)=\lambda^{0}+\Delta \lambda \mathrm{e}^{i \omega t}
$$

the distribution $I_{j}\left(\theta_{j}, \phi_{j}\right)$ must respond linearly in uniaxial symmetric fashion as ${ }^{5}$

$$
I_{j}\left(\theta_{j}, 0, t\right)=I_{j}^{0}\left(\theta_{j}, 0\right)+\Delta I_{j}^{*}\left(\theta_{j}, 0\right) \mathrm{e}^{i \omega t}
$$

where $\Delta I_{j}{ }^{*}\left(=I_{j}{ }^{\prime}+i \Delta I_{j}{ }^{\prime \prime}\right)$ is the dynamic complex amplitude of the X-ray diffraction intensity distribution, $\omega$, the angular velocity, and the superscript zero denotes the static component.

In analogy with eq 8 , the dynamic response of the orientation distribution function of the $j$-th crystal plane is given by

$$
\begin{aligned}
q_{j}\left(\zeta_{j}, 0, t\right) & =I_{j}\left(\theta_{j}, 0, t\right) / 4 \pi \int_{0}^{\pi / 2} I_{j}\left(\theta_{j}, 0, t\right) \sin \theta_{j} \mathrm{~d} \theta_{j} \\
& =\frac{I_{j}^{0}\left(\theta_{j}, 0\right)+\Delta I_{j}^{*}\left(\theta_{j}, 0\right) \mathrm{e}^{i \omega t}}{4 \pi \int_{0}^{\pi / 2} I_{j}^{0}\left(\theta_{j}, 0\right) \sin \theta_{j} \mathrm{~d} \theta_{j}+4 \pi \int_{0}^{\pi / 2} \Delta I_{j}^{*}\left(\theta_{j}, 0\right) \sin \theta_{j} \mathrm{~d} \theta_{j} \mathrm{e}^{i \omega t}}
\end{aligned}
$$


The Mechanical Dispersion and the Dynamic Crystal Orientation of Spherulitic PP and PB-1

The number of crystal planes per unit volume contributing to the $j$-th crystal diffraction intensity distribution must be constant if neither crystallization nor melting of the crystal grains occurs during the dynamic mechanical excitation. This postulate leads to

$$
\int_{0}^{\pi / 2} \Delta I_{j}^{*}\left(\theta_{j}, 0\right) \sin \theta_{j} \mathrm{~d} \theta_{j}=0
$$

and enables us to define the static and dynamic components of $q_{j}\left(\zeta_{j}, 0, t\right)$ as follows:

$$
\begin{gathered}
q_{j}{ }^{0}\left(\zeta_{j}, 0\right)=I_{j}{ }^{0}\left(\theta_{j}, 0\right) / 4 \pi \int_{0}^{\pi / 2} I_{j}{ }^{0}\left(\theta_{j}, 0\right) \sin \theta_{j} \mathrm{~d} \theta_{j} \\
\Delta q_{j}^{\prime}\left(\zeta_{j}, 0\right)=\Delta I_{j}^{\prime}\left(\theta_{j}, 0\right) / 4 \pi \int_{0}^{\pi / 2} I_{j}^{0}\left(\theta_{j}, 0\right) \sin \theta_{j} \mathrm{~d} \theta_{j} \\
\Delta q_{j}^{\prime \prime}\left(\zeta_{j}, 0\right)=\Delta I_{j}^{\prime \prime}\left(\theta_{j}, 0\right) / 4 \pi \int_{0}^{\pi / 2} I_{j}{ }^{0}\left(\theta_{j}, 0\right) \sin \theta_{j} \mathrm{~d} \theta_{j}
\end{gathered}
$$

where $\Delta q_{j}{ }^{\prime}\left(\zeta_{j}, 0\right)$ and $\Delta q_{j}{ }^{\prime \prime}\left(\zeta_{j}, 0\right)$ are, respectively, the real (in-phase) and imaginary (out-of-phase) components of the complex dynamic orientation function of the $j$-th crystal plane with respect to the dynamic bulk strain.

The dynamic response of the expansion coefficient $\alpha_{l 0}^{j}(t)$ can be defined as

$$
\alpha_{l 0}^{j}(t)=\alpha_{l 0}^{j^{0}}+\left(\Delta \alpha_{l 0}^{j^{\prime}}+i \Delta \alpha_{l 0}^{j^{\prime \prime}}\right) \mathrm{e}^{i \omega t}
$$

and its static and dynamic components can be further formulated from eq 6 as

$$
\begin{aligned}
\alpha_{l 0}^{j 0} & =2 \int_{0}^{1} q_{j}^{0}\left(\zeta_{j}, 0\right) \Pi_{l}\left(\zeta_{j}\right) \mathrm{d} \zeta_{j} \\
\Delta \alpha_{l 0}^{j \prime} & =2 \int_{0}^{1} \Delta q_{j}^{\prime}\left(\zeta_{j}, 0\right) \Pi_{l}\left(\zeta_{j}\right) \mathrm{d} \zeta_{j} \\
\Delta \alpha_{l 0}^{j \prime \prime} & =2 \int_{0}^{1} \Delta q_{j}^{\prime \prime}\left(\zeta_{j}, 0\right) \Pi_{l}\left(\zeta_{j}\right) \mathrm{d} \zeta_{j}
\end{aligned}
$$

When $l$ in eq 17 is fixed at $2, \alpha_{l 0}^{j^{0}}$ defines the second moment of the uniaxial orientation distribution function $q_{j}\left(\zeta_{j}, 0\right)$; i.e., $\alpha_{20}^{j}$, which allows Herman's uniaxial orientation factor to define as

$$
F_{20}^{j}=\frac{1}{2}\left(3\left\langle\cos ^{2} \theta_{j}\right\rangle-1\right)=\sqrt{2 / 5} 2 \pi \alpha_{20}^{j}
$$

The complex dynamic strain-orientation coefficient function of the reciprocal lattice vector of the $j$-th crystal plane, $C_{j}{ }^{*}\left(=C_{j}{ }^{\prime}+i C_{j}{ }^{\prime \prime}\right)$, may be defined as $^{17}$

$$
\begin{aligned}
C_{j}^{*}(i \omega) & =\Delta F_{20}^{j *} /\left.\Delta \lambda^{*}\right|_{\lambda=\lambda^{0}} \\
& =\sqrt{2 / 5} 2 \pi \Delta \alpha_{20}^{j *} /\left.\Delta \lambda\right|_{\lambda=\lambda^{0}}
\end{aligned}
$$

As in eq 16, the dynamic response of the coefficient of $w(\xi, 0, \eta)$ expanded the spherical harmonics, $A_{l 0 n}(t)$, can be defined as

$$
A_{l 0 n}(t)=A_{l 0 n}^{0}+\left(\Delta A_{l 0 n}^{\prime}+i \Delta A_{l 0 n}^{\prime \prime}\right) \mathrm{e}^{i \omega t}
$$

and eq 7 must be modified for the dynamic response as follows:

$$
\begin{aligned}
\alpha_{l 0}^{j 0}= & 2 \pi\left(\frac{2}{2 l+1}\right)^{1 / 2}\left[A_{l 00}^{0} \Pi_{l}\left(\cos \Theta_{j}\right)\right. \\
& \left.+2 \sum_{n=1}^{l} A_{l 0 n}^{0} \Pi_{l}{ }^{n}\left(\cos \Theta_{j}\right) \cos \left(n \Phi_{j}\right)\right] \\
\Delta \alpha_{l 0}^{j \prime}= & 2 \pi\left(\frac{1}{2 l+1}\right)^{1 / 2}\left[\Delta A_{l 00}^{\prime} \Pi_{l}\left(\cos \Theta_{j}\right)\right. \\
& \left.+2 \sum_{n=1}^{l} \Delta A_{l 0 n}^{\prime} \Pi_{l}{ }^{n}\left(\cos \Theta_{j}\right) \cos \left(n \Phi_{j}\right)\right] \\
\Delta \alpha_{l 0}^{j \prime}= & 2 \pi\left(\frac{2}{2 l+1}\right)^{1 / 2}\left[\Delta A_{l 00}^{\prime \prime} \Pi_{l}\left(\cos \Theta_{j}\right)\right. \\
& \left.+2 \sum_{n=1}^{l} \Delta A_{l 0 n}^{\prime \prime} \Pi_{l}{ }^{n}\left(\cos \Theta_{j}\right) \cos \left(n \Phi_{j}\right)\right]
\end{aligned}
$$

Furthermore, the dynamic response of the orientation distribution function of the crystal grains $w(\xi, 0, \eta, t)$ can be defined as

$$
\begin{aligned}
w(\xi, 0, \eta, t)= & w^{0}(\xi, 0, \eta)+\left[\Delta w^{\prime}(\xi, 0, \eta)\right. \\
& \left.+i \Delta w^{\prime \prime}(\xi, 0, \eta)\right] \mathrm{e}^{i \omega t}
\end{aligned}
$$

where $w^{0}(\xi, 0, \eta)$ is the static component at $\lambda=\lambda^{0}$, and $\Delta w^{\prime}(\xi, 0, \eta)$ and $\Delta w^{\prime \prime}(\xi, 0, \eta)$ are, respectively, the real and imaginary parts of the complex dynamic orientation distribution function of the crystal grains. Each part can be obtained from eq 3 as

$$
\begin{aligned}
w^{0}(\xi, 0, \eta)= & \sum_{l=0}^{\infty} A_{l 00}^{0} \Pi_{l}(\xi) \\
& +2 \sum_{l=2}^{\infty} \sum_{n=1}^{l} A_{l 0 n}^{0} \Pi_{l}{ }^{n}(\xi) \cos (n \eta) \\
\Delta w^{\prime}(\xi, 0, \eta)= & \sum_{l=0}^{\infty} \Delta A_{l 00}^{\prime} \Pi_{l}(\xi) \\
& +2 \sum_{l=2}^{\infty} \sum_{n=1}^{l} \Delta A_{l 0 n}^{\prime} \Pi_{l}{ }^{n}(\xi) \cos (n \eta)
\end{aligned}
$$




\section{K. FujiTA et al.}

$$
\begin{aligned}
\Delta w^{\prime \prime}(\xi, 0, \eta)= & \sum_{l=0}^{\infty} \Delta A_{l 00}^{\prime \prime} \Pi_{l}(\xi) \\
& +2 \sum_{l=2}^{\infty} \sum_{n=1}^{l} \Delta A_{l 0 n}^{\prime \prime} \Pi_{l}{ }^{n}(\xi) \cos (n \eta)
\end{aligned}
$$

As in the determination of $w(\xi, 0, \eta)$ from $q_{j}\left(\zeta_{j}, 0\right)$, the static and dynamic components, $w^{0}(\xi, 0, \eta), \Delta w^{\prime}(\xi, 0, \eta)$, and $\Delta w^{\prime \prime}(\xi, 0, \eta)$ can be obtained from $q_{j}{ }^{0}\left(\zeta_{j}, 0\right), \Delta q_{j}{ }^{\prime}\left(\zeta_{j}, 0\right)$ and $\Delta q_{j}{ }^{\prime \prime}\left(\zeta_{j}, 0\right)$ and evaluating $\alpha_{l 0}^{j^{0}}, \Delta \alpha_{l 0}^{j^{\prime}}$ and $\Delta \alpha_{l 0}^{j^{\prime \prime}}$ and $A_{l 0 n}^{0}, \Delta A_{l 0 n}^{\prime}$, and $\Delta A_{l 0 n}^{\prime \prime}$ using the respective equations from eq 16 through 29 , all under the approximation that the series are truncated at $l=(j-1)$ and $l=6(j-1)$ for polypropylene and polybutene-1, respectively.

\section{EXPERIMENTAL RESULTS AND DISCUSSION}

Uniaxial Deformation Mechanism of Spherulitic Crystalline Texture as Observed from the Change in $w(\xi, 0, \eta)$ with Extension Ratio

Figures 5 and 6 show the changes in the birefringence of the it-PP B-200(A) and it-PB-1 M801N(A) specimens with tensile strain at three different temperatures, 25,60 , and $100^{\circ} \mathrm{C}$. As can be seen from the figures, both specimens exhibit an increase in birefringence in a negative direction at the initial stage of stretching and then in a positive direction with an increase in stretching. Thus the birefringence passes a maximum value negative in sign. With stretching temperature, this behavior of birefringence becomes more pronounced.

As has been discussed by Sasaguri et al., ${ }^{18-20}$ this birefringence behavior may be explained as a result of the superposition of at least two orientation mechanisms of different structural units having different intrinsic birefringence in sign; i.e., crystal lamellae having a negative intrinsic birefringence and crystal grains within the lamella having a positive intrinsic birefringence. In the undeformed state the spherulite consisting of the crystal lamellae exhibits the so-called negative spherulite, as indicated in connection with the $V_{\mathrm{v}}$ light scattering in Figure 3. At the initial stage of stretching, the uniaxial deformation of the spherulite must be associated with the lamellar orientation to give a negative birefringence in bulk. At the intermediate stage of stretching where the birefringence attains a maximum negative value, the orientation of the

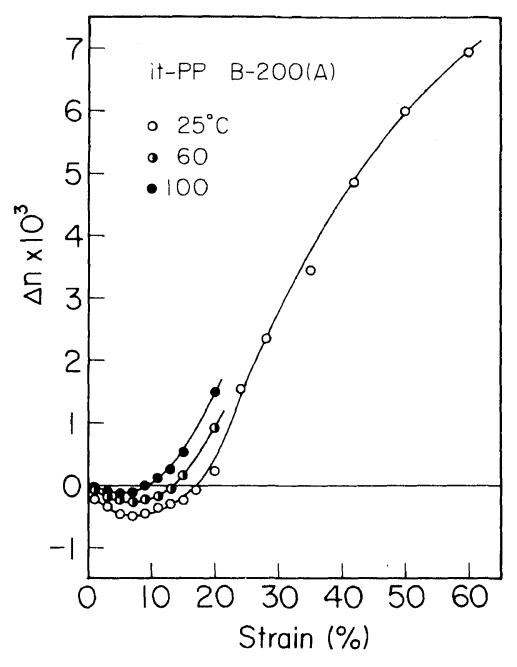

Figure 5. Change in the birefringence of it-PP B200(A) specimen with tensile strain imposed at three different temperatures, 25,60 , and $100^{\circ} \mathrm{C}$.

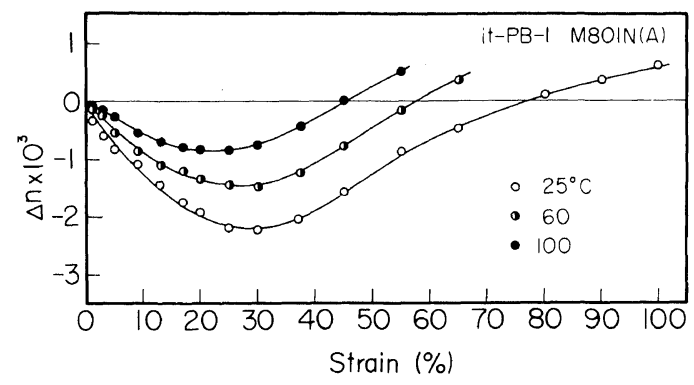

Figure 6. Change in the birefringence of it-PB-1 M801N(A) specimen with tensile strain imposed at three different temperatures, 25,60 , and $100^{\circ} \mathrm{C}$.

crystal lamellae must be accompanied by reorientation of the crystal grains, causing the orientation of the crystal grains having a positive intrinsic birefringence toward the stretching direction and resulting in a positive birefringence in bulk.

Figures 7 and 8 compare the observed orientation distribution functions, $q_{j}\left(\zeta_{j}, 0\right)$, of the $j$-th crystal planes with those calculated for the respective crystal planes, at given extension ratios for the it-PP B200(A) and it-PB-1 M801N(A) specimens, respectively, both stretched at a fixed temperature of $60^{\circ} \mathrm{C}$. In this case, $\alpha_{l 0}^{j}$ was first calculated from eq 6 using $q_{j}\left(\zeta_{j}, 0\right)$ observed for the $j$-th crystal plane, and $A_{l 0 n}$ was determined from eq 7 by solving a set of simultaneous equations in eq 7 varying $j$ up to 7 
The Mechanical Dispersion and the Dynamic Crystal Orientation of Spherulitic PP and PB-1

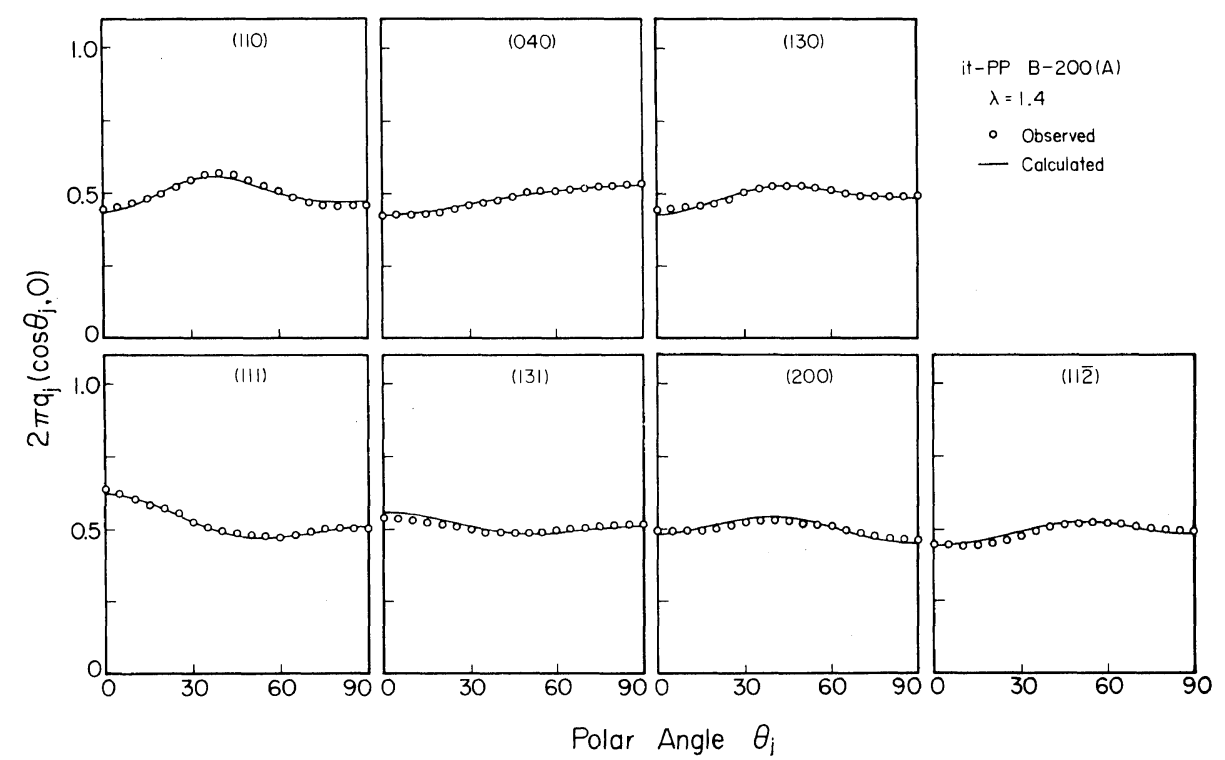

Figure 7. Comparison of observed uniaxial orientation distribution functions $q_{j}\left(\zeta_{j}, 0\right)$ (open circles) for 7 different $j$-th crystal planes for the it-PP B-200(A) specimen with those calculated (solid lines) at a given extension ratio of 1.4 .

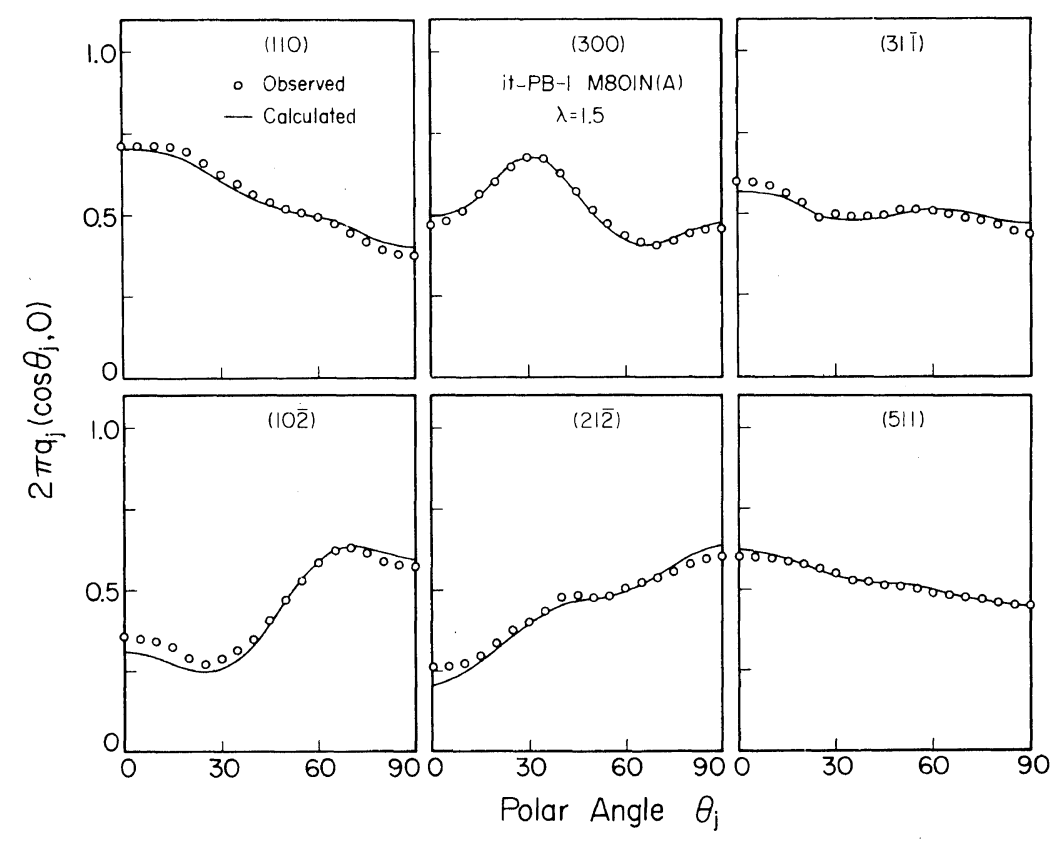

Figure 8. Comparison of observed uniaxial orientation distribution functions $q_{j}\left(\zeta_{j}, 0\right)$ (open circles) for 6 different $j$-th crystal planes for the it-PB-1 M801N(A) specimen with those calculated (solid lines) at a given extension ratio of 1.5 . 


\section{K. Fujita et al.}

for polypropylene and 6 for polybutene-1. Then, $\alpha_{10}^{j}$ was recalculated from eq 7 by using $A_{l 0 n}$ thus determined, and further, $q_{j}\left(\zeta_{j}, 0\right)$ was calculated from eq 4 with a finite series of expansion up to order of $l=6$ th and $=30$ th for the respective polymers. As can be seen from the figures, fairly good agreement between the observed and calculated distribution functions can be achieved even for crystal planes less accurately measured due to faint diffraction intensities, such as the (200) and (112) for polypropylene and the (311) and (511) crystal planes for polybutene-1. This indicates a high reliability of the $A_{l 0 n}$ thus determined.

Figures 9 and 10 show the changes in the uniaxial orientation distribution frunction $w(\xi, 0, \eta)$ with the extension ratio for the respective specimen stretched at $60^{\circ} \mathrm{C}$. These functions were calculated from eq 3 by retaining $A_{l 0 n}$ up to the 6th and 30th orders of $l$ for it-PP B-200(A) and it-PB-1 M801N(A), respectively. Figure 9 for polypropylene shows that a populous region appears at $\theta \simeq 90^{\circ}$ and $\eta=0^{\circ}$ at the initial stage of stretching and moves to a smaller angle $\theta$, with $\eta$ remaining at $0^{\circ}$, accompanied by the development of an additional populous region at $\theta \simeq 0^{\circ}$ at the intermediate stage of stretching. At the final stage of stretching up to $\lambda=1.8$, the populous region is concentrated at $\theta=0^{\circ}$.

In contrast to a four-fold symmetry of the orientation distribution function for the polypropylene specimen, the distribution function of the polybutene-1 specimen exhibits a six-fold symmetry, as seen in Figure 10. This is due to the hexagonal symmetry of the Cartesian coordinate system 0 $u_{1} u_{2} u_{3}$ fixed within the crystal. Consequently, the orientation distribution function of the polybutene1 specimen in a range of $\eta$ from 0 to $30^{\circ}$ corresponds to that of the polypropylene in a range of $\eta$ from 0 to $90^{\circ}$. Thus the crystal orientation behavior of the polybutene-1 specimen is very similar to that of the polypropylene specimen, except for a populous region remaining at $\theta \simeq 60^{\circ}$ and $\eta=0^{\circ}$, even at the final stage of stretching to an extension ratio as high as 2.0 beyond which macroscopic-necking of the specimen occurs.

As has been already investigated in the previous paper, ${ }^{1,3}$ the uniaxial orientation behavior of spherulitic high-density polyethylene can be characterized in terms of the change in $w(\xi, 0, \eta)$ as follows: two populous regions appear at the initial stage of stretching: one, though broad and somewhat ob- scure, at $\theta \simeq 90^{\circ}$ and $\eta \simeq 90^{\circ}$, and the other, at $\theta \simeq 20^{\circ}$ and $\eta=0^{\circ}$. It seems that the former populous region moves to smaller $\theta$ with $\eta$ unchanged at $90^{\circ}$ and becomes much prominent, while the latter populous region remains at the same position and becomes somewhat clearer, as the extension ratio increases. Here, the Cartesian coordinate system $0-u_{1} u_{2} u_{3}$ is fixed within the orthorhombic polyethylene crystal in such a way that the $u_{1}, u_{2}$, $u_{3}$ axes are parallel to the $a-, b$-, and $c$-axes of the crystal.

Figure 11 shows the orientation distribution function, $w(\xi, 0, \eta)$, calculated from a uniaxial deformation model of a spherulite with the extension ratio of the spherulite fixed at 1.2 and rather extreme values, assigned to the model parameter. These choices were made to combine the affine lamellar orientation with the reorientation of the crystal grains within the orienting lamellae according to the following five types of orientation mechanisms ${ }^{1,3}$ :

1) Type A orientation: preferential $a^{*}$-axis orientation associated with random rotation of the crystal grains about the $a^{*}$-axes, corresponding to the preferential orientation of twisted lamellae for polypropylene and polybutene- 1 spherulites, not associated with any rotation of the crystal grains within the lamellae.

2) Type B orientation: preferential $b$-axis orientation associated with random rotation of the crystal grains about the $b$-axes, corresponding to the preferential orientation of twisted lamellae for polyethylene spherulites, not associated with any rotation of the crystal grains within the lamellae.

3) Type $\mathrm{Ca}$ orientation: preferential $c$-axis orientation associated with rotation of the crystal grains about their own $a$ - or $a^{*}$-axis, causing the crystal $c$-axes (molecular axes) to orient toward the stretching direction.

4) Type $\mathrm{Cb}$ orientation: preferential $c$-axis orientation associated with rotation of the crystal grains about their own $b$-axes, causing the crystal $c$ axes to orient toward the stretching direction.

5) Type $\mathrm{Cc}$ orientation: preferential $c$-axis orientation associated with random rotation of the crystal grains about the $c$-axes causing the crystal $c$ axes to orient themselves in the stretching direction and lead to the so-called fiber structure.

The uniaxial deformation mechanism of spherulites has already been formulated on the basis of 
The Mechanical Dispersion and the Dynamic Crystal Orientation of Spherulitic PP and PB-1

$4 \pi^{2} w(\xi, 0, \eta)$ it-PP B-200(A)
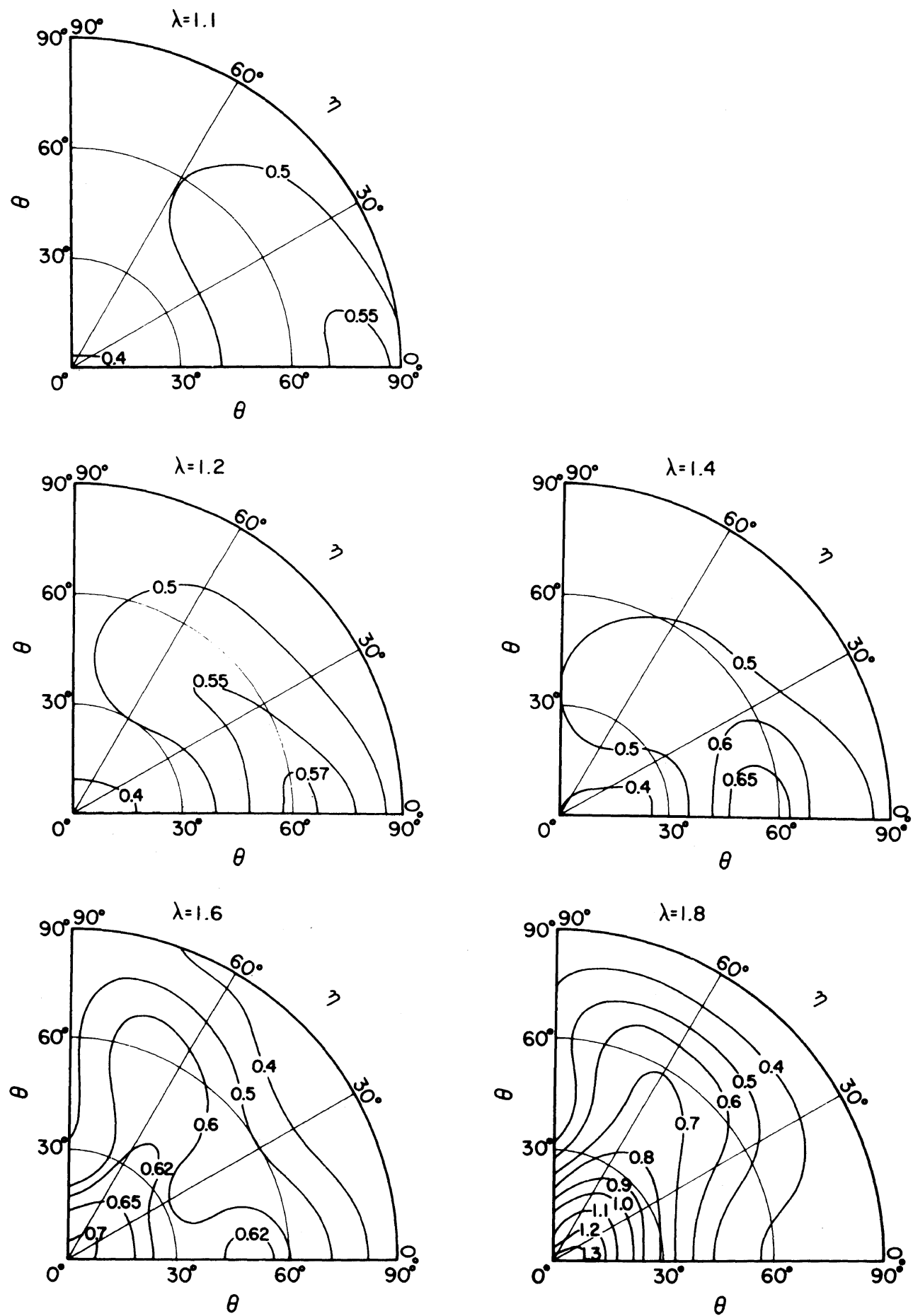

Figure 9. Change in the uniaxial orientation distribution function of crystal grains, $w(\xi, 0, \eta)$ with an extension ratio up to 1.8 for the it-PP B-200(A) specimen at $60^{\circ} \mathrm{C}$. 
K. Fujita et al.

$4 \pi^{2} w(\xi, 0, \eta)$ it-PB-1 M $801 \mathrm{~N}(\mathrm{~A})$
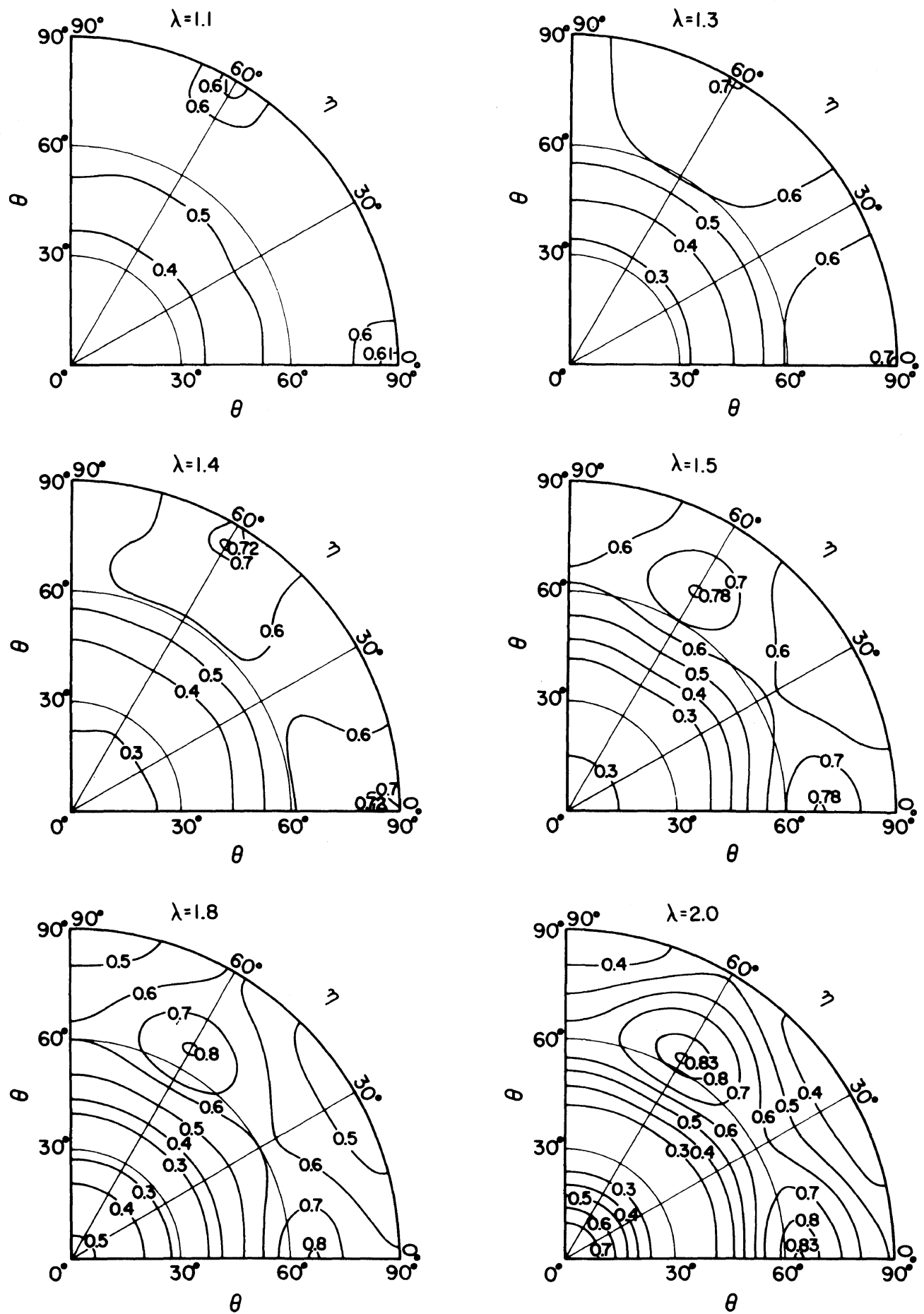

Figure 10. Change in the uniaxial orientation distribution function of crystal grains, $w(\xi, 0, \eta)$ with an extension ratio up to 2.0 for the it-PB-1 M $801 \mathrm{~N}(\mathrm{~A})$ specimen at $60^{\circ} \mathrm{C}$. 
The Mechanical Dispersion and the Dynamic Crystal Orientation of Spherulitic PP and PB-1

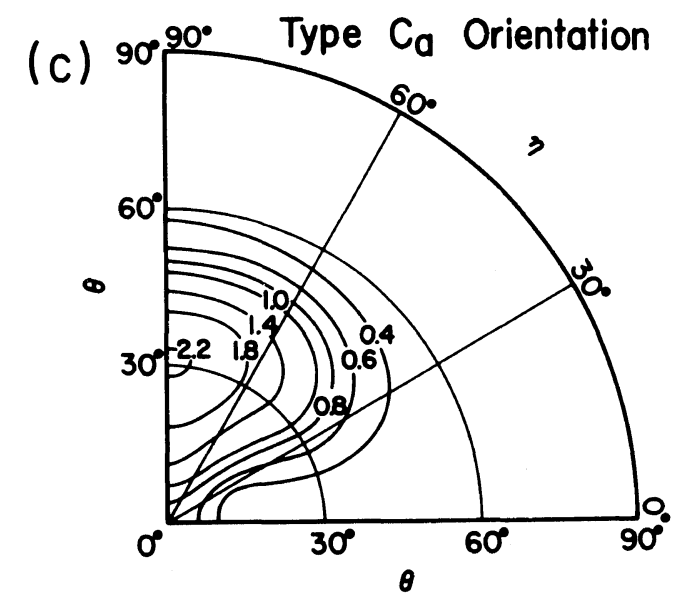

(a)

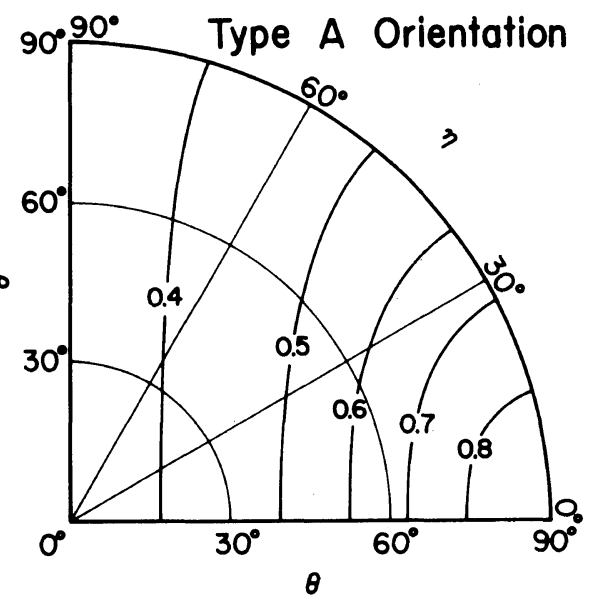

(b)

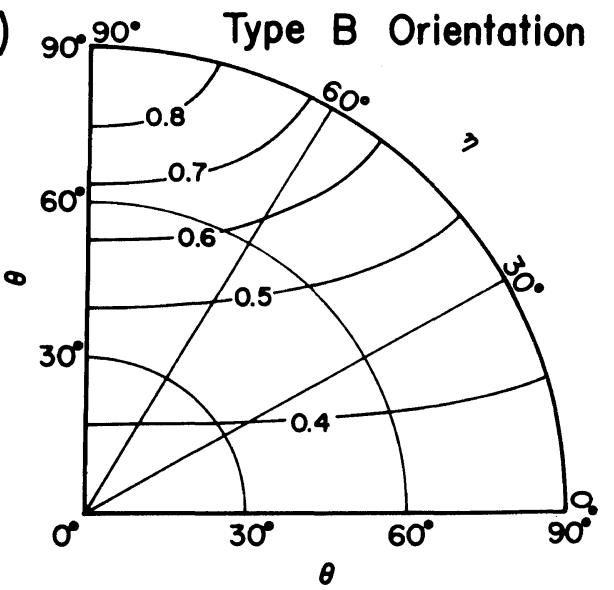

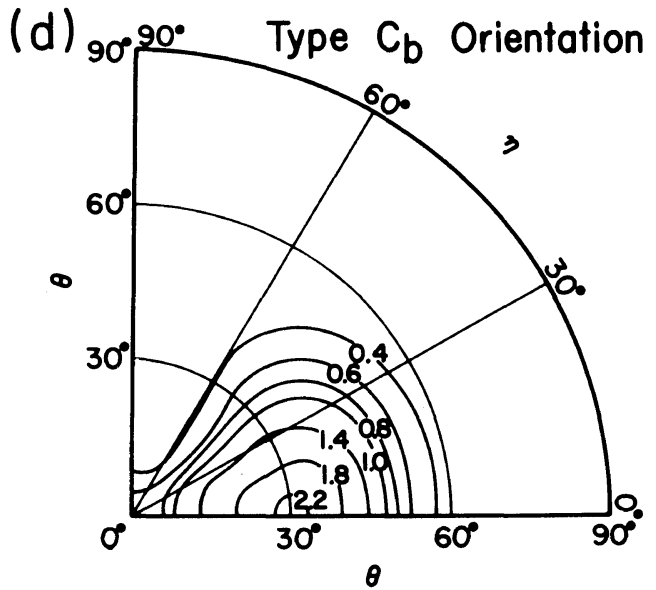

(e)

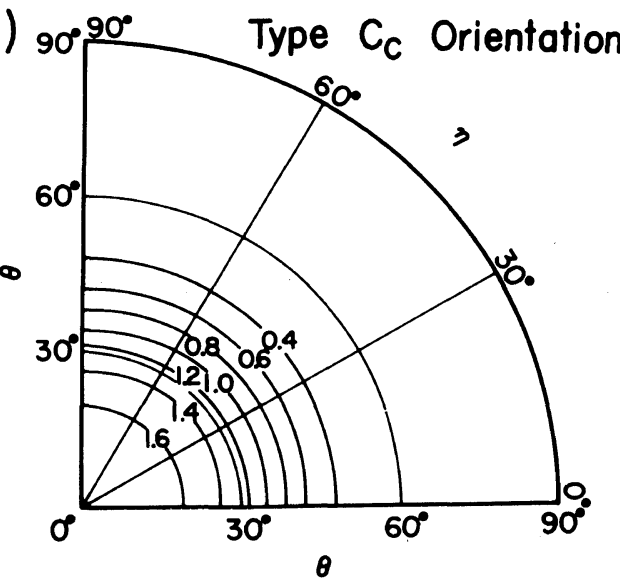

Figure 11. Uniaxial orientation distribution functions of the crystal grains, $w(\xi, 0, \eta)$ calculated from a spherulite deformation model by fixing the extension ratio at 1.2 and the model parameters at rather extreme values, so as to combine the lamellar orientation in an affine fashion with the reorientation of the crystal grains within the orienting lamellae in the specific fashion of the five types of orientation mechanisms: (a) type $\mathrm{A}$; (b) type $\mathrm{B}$; (c) type $\mathrm{Ca}$; (d) type $\mathrm{Cb}$; and (e) type $\mathrm{Cc}$. 
K. Fujita et al.
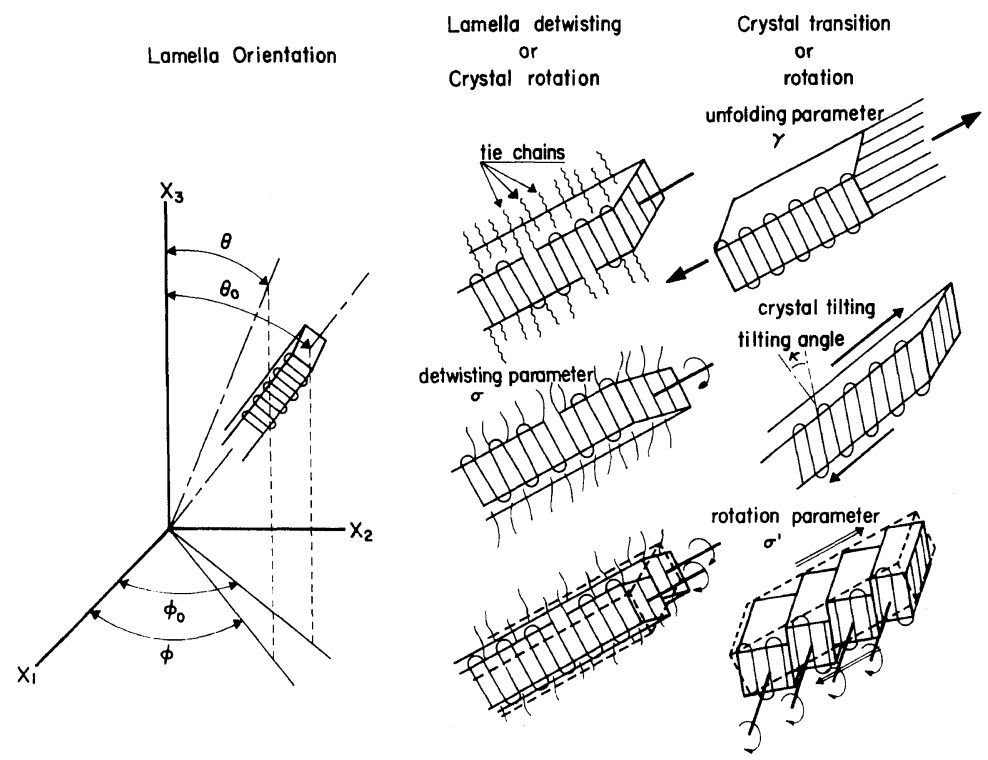

Figure 12. Schematic diagram demonstrating three types of intra-lamellar shearing mechanisms: lamellar detwisting, lamellar tilting, and lamellar unfolding (micronecking) mechanisms.

the above model. ${ }^{1,3}$ However, we discuss here the idea of the formulation qualitatively. Type $\mathrm{A}$ and type $\mathrm{B}$ orientations are formulated from the spherulitic crystalline textures of poly-alpha-olefins, to represent the lamellar orientation as a rigid body. Type $\mathrm{Ca}, \mathrm{Cb}$, and $\mathrm{Cc}$ orientations are formulated for the polyethylene spherulite to represent intralamellar shearing, corresponding respectively to the tilting, detwisting, and unfolding (micronecking) of the lamellae illustrated in Figure 12. For the polypropylene and polybutene- 1 spherulites, however, these representations for type $\mathrm{Ca}$ and $\mathrm{Cb}$ orientations must be reversed, because the lamellar axis is parallel to the $a^{*}$-axis, and not to the $b$-axis, as in polyethylene spherulites. By taking into account the topological and morphological features, the degrees of type $\mathrm{Ca}$ and $\mathrm{Cb}$ orientations were formulated as a function of lamellar orientation in such a way that the former and latter orientations for polyethylene spherulites are accentuated at the polar and equatorial zones of the uniaxially deformed spherulites, and vice versa for polypropylene and polybutene-1 spherulites.

Comparing the experimental results of $w(\xi, 0, \eta)$ for the three kinds of poly-alpha-olefins mentioned above with the calculated results in Figure 11, the spherulite deformation mechanism can be characterized for the respective polymers as follows:
1) For the polyethylene specimen, type $B$ and $\mathrm{Cb}$ orientations occur at the initial stage of stretching; i.e., the lamellar orientation associated with lamellar detwisting mostly occurs at the equatorial zone of the uniaxial deformed spherulites. The type $\mathrm{B}$ orientation is followed by type $\mathrm{Ca}$ orientation with a great rate at the intermediate stage of stretching; i.e., the lamellar orientation associated with the lamellar tilting occurs mostly at the polar zone of the spherulites. At the final stage of stretching, type $\mathrm{Ca}$ and $\mathrm{Cb}$ orientations, especially the former, tend to orient the crystal $c$-axes of the crystal grains further toward the stretching direction.

2) For the polypropylene specimen, type A orientation and probably type $\mathrm{Cb}$ orientation associated to some extent with the lamellar tilting occurs mostly in the polar zone of the uniaxially deformed spherulites. Type A orientation is followed by the type $\mathrm{Cb}$ orientation in a great rate to yield type $\mathrm{Cc}$ orientation at the intermediate stage of stretching; i.e., the lamellar orientation associated with the lamellar tilting and a partial lamellar micronecking also occurs mostly at the polar zone of the spherulites. At the final stage of stretching, type $\mathrm{Cb}$ orientation further advances and is completely changed to type $\mathrm{Cc}$ orientation; i.e., the disintegration of the spherulitic crystalline 
texture to the so-called fiber structure takes place. It should be emphasized that type $\mathrm{Ca}$ orientation, corresponding to the lamellar detwisting, seldom occurs in the polypropylene specimen in contrast to the polyethylene specimen.

3) For the polybutene-1 specimen, the spherulite deformation mechanism is very similar to that of the polypropylene specimen. The only exception is that type $\mathrm{Cb}$ orientation is not completely changed to type $\mathrm{Cc}$ orientation, even at the final stage of stretching where the bulk specimen may undergo macroscopic necking. It may be again emphasized that type $\mathrm{Ca}$ orientation, also corresponding to lamellar detwisting, seldom develops in the polybutene-1 specimen in contrast to the poly- ethylene specimen.

It is summarized, at least qualitatively, that the uniaxial deformation of spherulitic crystalline texture of poly-alpha-olefins can be characterized by the affine orientation of crystal lamellar and is associated with three kinds of intra-lamellar shearing mechanisms in different, i.e., lamellar tilting, detwisting, and micronecking, extent depending on the location within the spherulites (see Figure 13) and the degree of extension of the spherulites.

For polyethylene spherulites, the lamellar detwisting occurs mostly at the equatorial zone of the uniaxially deformed spherulites at the initial stage of the spherulite deformation, and then the lamellar tilting occurs mostly at the polar zone of the

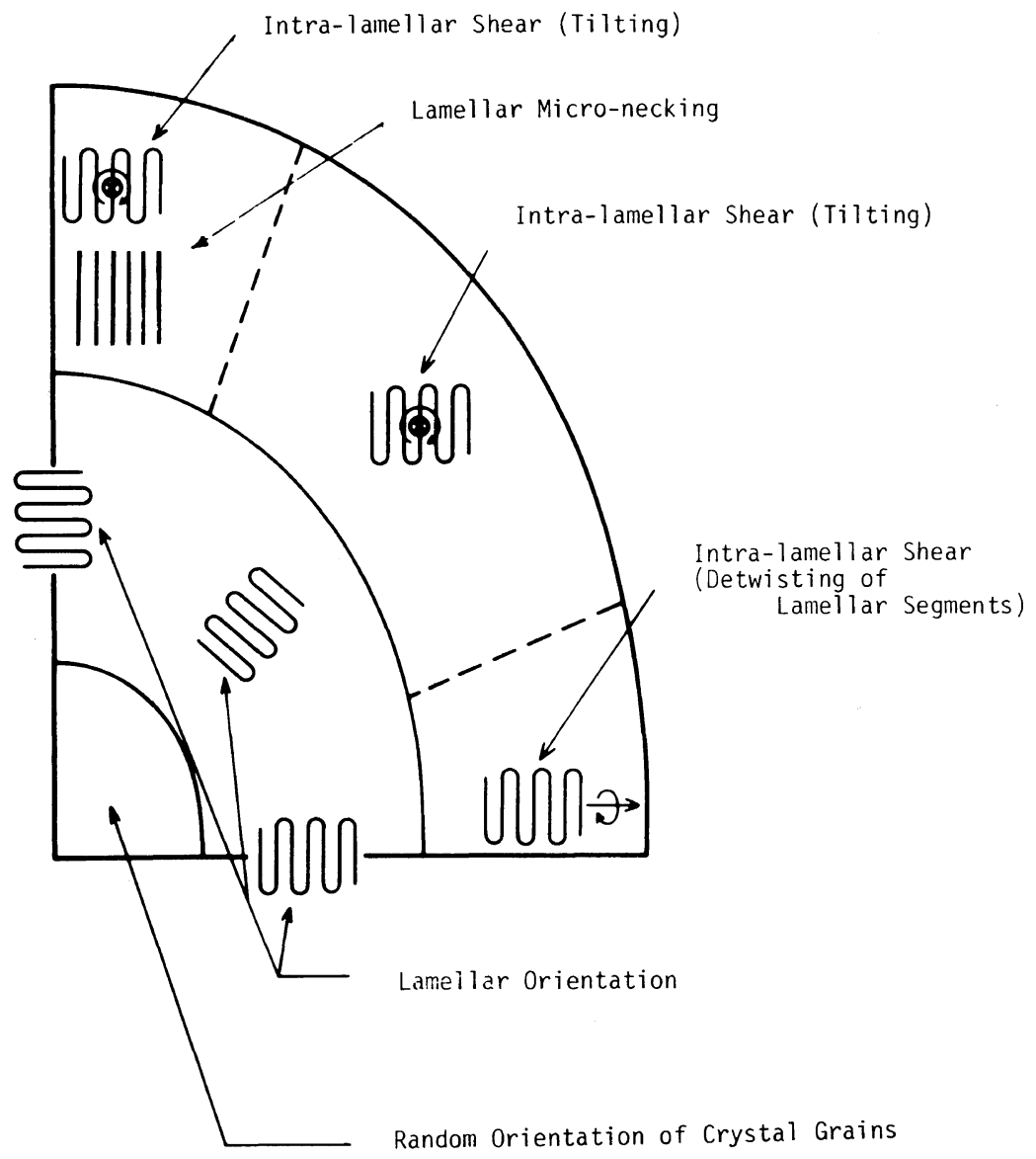

Figure 13. Schematic diagram of uniaxial deformation mechanism of spherulitic crystalline texture of poly-alpha-olefins, combining the lamellar orientation with the three types of intra-lamellar shearing mechanisms located at particular positions within the spherulites. 


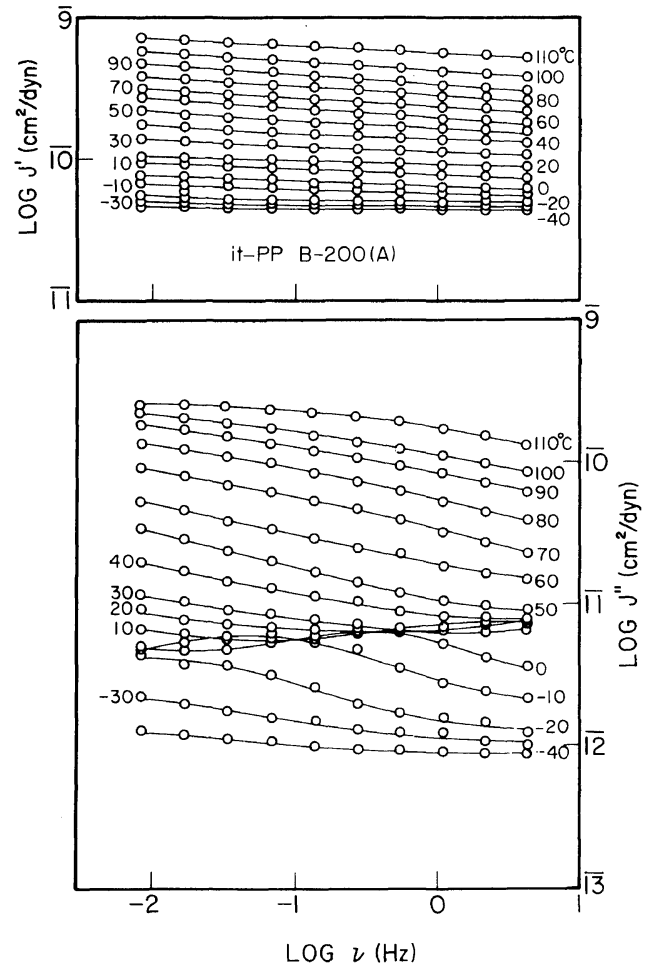

Figure 14. Temperature dependences of the storage and loss compliance functions of the it-PP B-200(A) specimen.

spherulites at a rate much greater than that for the lamellar detwisting. For polypropylene and polybutene- 1 spherulites, on the other hand, the lamellar tilting occurs mostly at the polar zone of the uniaxially deformed spherulites even at the initial stage of stretching, and is followed by micronecking of the lamellae; i.e., the distintegration of the spherulitic texture to the fiber structure.

\section{Dynamic Mechanical and Optical Dispersions}

Figures 14 and 15 show the temperature dependence of the storage and loss compliance functions of the it-PP B-200(A) and it-PB-1 M801N(A) specimens, respectively, over a temperature range from -40 to about $100^{\circ} \mathrm{C}$. The storage compliance reaches a minimum of about $4 \times 10^{-11} \mathrm{~cm}^{2} \mathrm{dyn}^{-1}$ at the lowest temperature of $-40^{\circ} \mathrm{C}$, but increases gradually with decreasing frequency at elevated temperatures, except near $0^{\circ} \mathrm{C}$ where the storage compliance increases rather remarkably with decreasing frequency for the it-PB-1 M801N(A) speci-

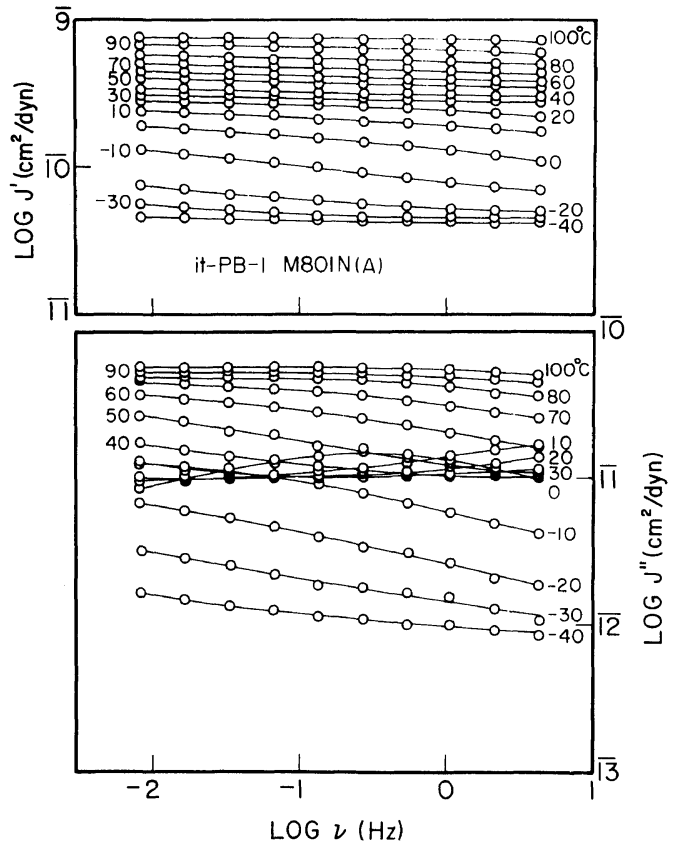

Figure 15. Temperature dependences of the storage and loss compliance functions of the it-PB-1 M801N(A) specimen.

men. The loss compliance increases remarkably with decreasing frequency and exhibits two maxima, a distinct one at a temperature around $0^{\circ} \mathrm{C}$ and another at the highest temperature, for both specimens. In agreement with the previous studies on spherulitic high-density polyethylene, ${ }^{3,21}$ the conventional frequency-temperature superposition by simple horizontal shifting of the compliance functions along the logarithmic frequency axis was not valid. Thus, some vertical shifts of the functions were necessary for obtaining well-superposed master curves.

The use of the vertical shift in conjunction with the horizontal shift in the process of superposition is usually arbitrary. To minimize this arbitrariness, the storage and loss compliance functions superposition simultaneously in such a way that the resulting shift factors could give the best results for both function. In Figures 16 and 17 are shown the superposed master curves so obtained for it-PP B200(A) and it-PB-1 M801N(A), with the reference temperatures chosen as 30 and $-20^{\circ} \mathrm{C}$, respectively. It can be seen that the frequency dispersion of the compliance functions is unusually broad, but, in 
The Mechanical Dispersion and the Dynamic Crystal Orientation of Spherulitic PP and PB-1

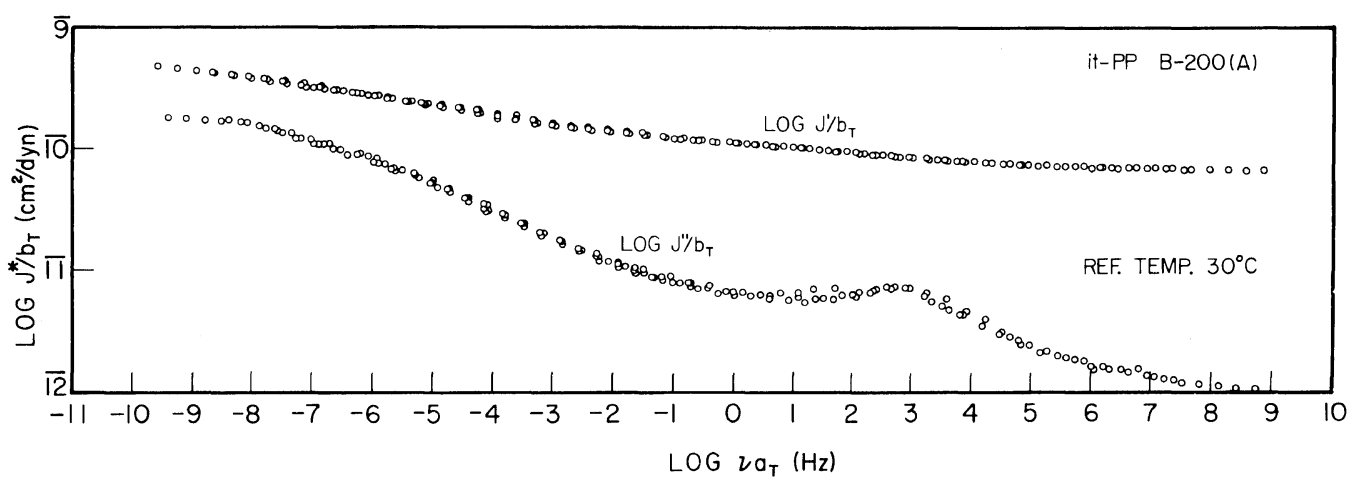

Figure 16. Superposed master curves of the storage and loss compliance functions of the it-PP B-200(A) specimen reduced to a reference temperature of $30^{\circ} \mathrm{C}$.

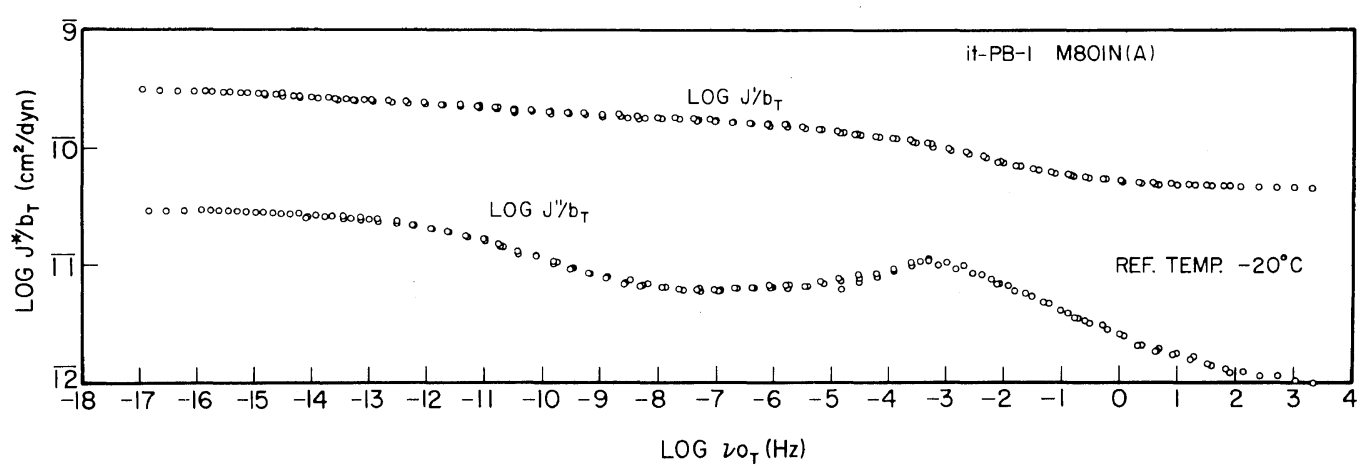

Figure 17. Superposed master curves of the storage and loss compliance functions of the it-PB-1 $\mathrm{M} 801 \mathrm{~N}(\mathrm{~A})$ specimen reduced to a reference temperature of $-20^{\circ} \mathrm{C}$.

contrast to the master curves of spherulitic highdensity polyethylene, ${ }^{21}$ the loss compliance function exhibits clearly an additional dispersion at a relatively high frequency as the specimen changes from polyethylene to polypropylene and polybutene-1.

In Figures 18 and 19, Arrhenius plots in which the logarithm of horizontal shift factor, $\log a_{\mathrm{T}}$, is plotted against the reciprocal absolute temperature are shown together with the temperature dependence of the vertical shift factor, $\log b_{\mathrm{T}}$ for it-PP B200(A) and it-PB-1 M801N(A), respectively. These plots for both specimens can be represented by two distinct straight lines. The activation energies of the corresponding retardation processes were estimated to be 37.7 and $47.4 \mathrm{kcal} \mathrm{mol}^{-1}$ and 42.3 and 60.4 $\mathrm{kcal} \mathrm{mol}^{-1}$, respectively, for the polypropylene and polybutene-1 specimens. These values and the associated temperature regions suggest the $\beta$ and $\alpha$ processes of these polymers.
Although the mechanical retardation or the relaxation processes of these polymers have been studied less than those of polyethylene, the activation energies of the $\beta$ and $\alpha$ retardation processes obtained here are in fairly good agreement with the literature values ${ }^{22-30}$; for example, Wada obtained about 40 and $60 \mathrm{kcal} \mathrm{mol}^{-1}{ }^{24}$ from the dielectric relaxation of polypropylene. However, these energies differ from the extremely large value of 170

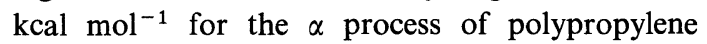
reported by Hara et al. ${ }^{26}$ By comparing the above results for polypropylene and polybutene- 1 with that for polyethylene, ${ }^{21}$ it may be emphasized that there does not seem to be any retardation process corresponding to the $\alpha_{2}$ mechanical dispersion of polyethylene for the polypropylene and polybutene1 specimens. The temperature dependence of the vertical shift factor, $\log b_{\mathrm{T}}$, is shown in Figures 18 and 19 for the respective polymers. This tempera- 


\section{K. FuJita et al.}

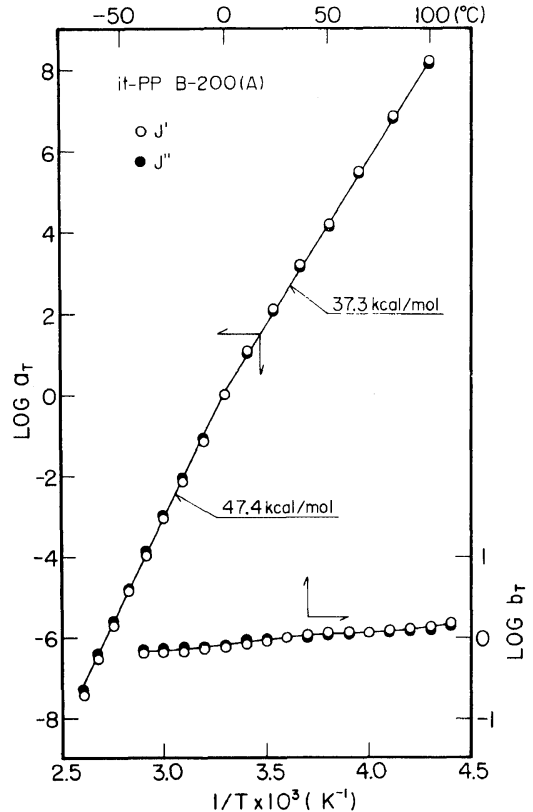

Figure 18. Arrhenius plots of the horizontal shift factor $\log a_{\mathrm{T}} v s$. reciprocal absolute temperature together with the temperature dependence of the vertical shift factor $\log b_{\mathrm{T}}$, both for composing the master curves in Figure 16 for the it-PP B-200(A) specimen.

ture dependence is not appreciable for either specimen over the range of temperature covered, when compared with the rather strong dependence for polyethylene at high temperature probably due to activation of the $\alpha_{2}$ mechanical retardation process. $^{21}$

The dynamic birefringence data obtained with the dynamic mechanical data were analyzed in terms of the real and imaginary components of the complex dynamic stress-optical coefficient function $M^{*}$ $\left(=M^{\prime}-i M^{\prime \prime}\right)$. This function is defined as the derivative of the complex dynamic amplitude of birefringence with respect to that of bulk stress. The real and imaginary components, $M^{\prime}$ and $M^{\prime \prime}$, are plotted in Figures 20 and 21 for the it-PP B200(A) and it-PB-1 M801N(A) specimens, respectively as functions of temperature over the range from -40 to about $100^{\circ} \mathrm{C}$. It should be noted that both real and imaginary components are negative in sign, revealing that the dynamic birefringence responds with negative amplitude and behind the dynamic bulk stress in the phase relation.

As can be seen in the figures, the real component

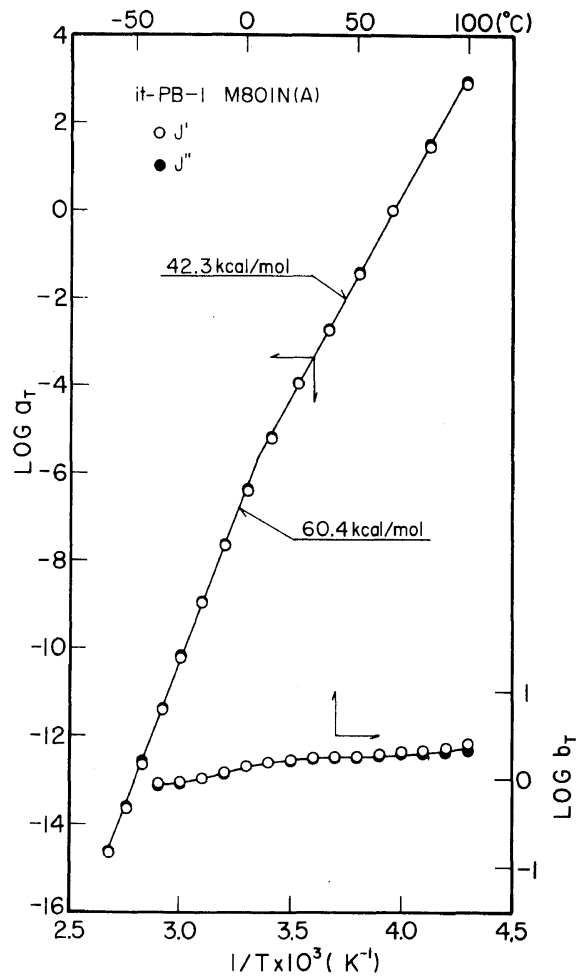

Figure 19. Arrhenius plots of the horizontal shift factor $\log a_{\mathrm{T}} v s$. reciprocal absolute temperature together with the temperature dependence of the vertical shift factor $\log b_{\mathrm{T}}$, both for composing the master curves in Figure 17 for the it-PB-1 M801N(A) specimen.

$-M^{\prime}$ reaches a minimum of about $-3 \times 10^{-13} \mathrm{~cm}^{2}$ $\mathrm{dyn}^{-1}$ at the lowest temperature of $-40^{\circ} \mathrm{C}$, but increases gradually with decreasing frequency at elevated temperatures except at temperatures near $0^{\circ} \mathrm{C}$ at which the real component exhibits a distinct frequency dispersion for either specimen. The imaginary component $-M^{\prime \prime}$ increases remarkably with decreasing frequency, exhibiting two maxima: one distinct at a temperature near $0^{\circ} \mathrm{C}$ and another being lenient at the highest temperature, for either specimen. As recognizable from these results, especially those of the imaginary components, the conventional frequency-temperature superposition is not valid, and a vertical shift is needed to obtain well superposed master curves of the functions.

Figures 22 and 23 show the master curves of the real and imaginary components of the complex dynamic stress-optical coefficient functions for it-PP B-200(A) and it-PB-1 M801N(A), respectively 
The Mechanical Dispersion and the Dynamic Crystal Orientation of Spherulitic PP and PB-1

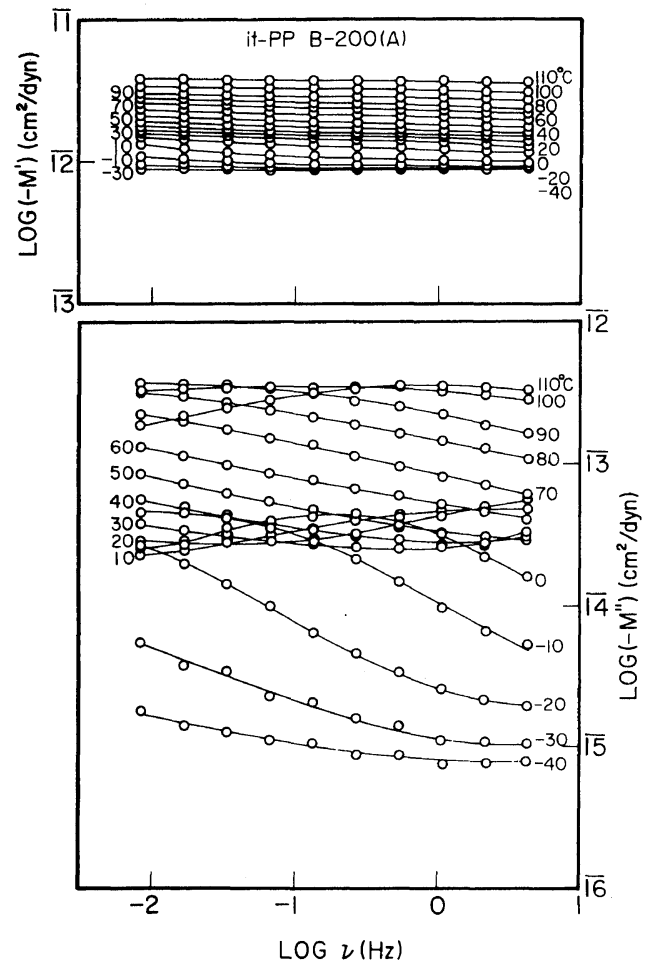

Figure 20. Temperature dependences of real and imaginary components of the complex dynamic stress-optical coefficient function of the it-PP B-200(A) specimen.

where the reference temperature is chosen as 30 and $-20^{\circ} \mathrm{C}$. For both real and imaginary components some vertical and horizontal shift factors were used to minimize the arbitrariness of the superposition, as in the case of the mechanical data. It can be seen that the frequency dispersion of the complex dynamic stress-optical coefficient function, especially the real component, is unusually broad, but in very contrast to the master curves of spherulitic highdensity polyethylene ${ }^{21}$ the imaginary component exhibits more clearly an additional dispersion at relatively high frequencies as the specimen changes from polyethylene to polypropylene and polybutene-1.

Figures 24 and 25 show the temperature dependence of $\log o_{\mathrm{T}}$ and $\log q_{\mathrm{T}}$, for the respective specimens. Two linear regions appear in the Arrhenius plots of $o_{\mathrm{T}}$ and the corresponding activation energies 36.8 and $50.7 \mathrm{kcal} \mathrm{mol}^{-1}$ and 45.2 and $64.1 \mathrm{kcal} \mathrm{mol}^{-1}$ for the respective specimens. These values and the associated temperature regions

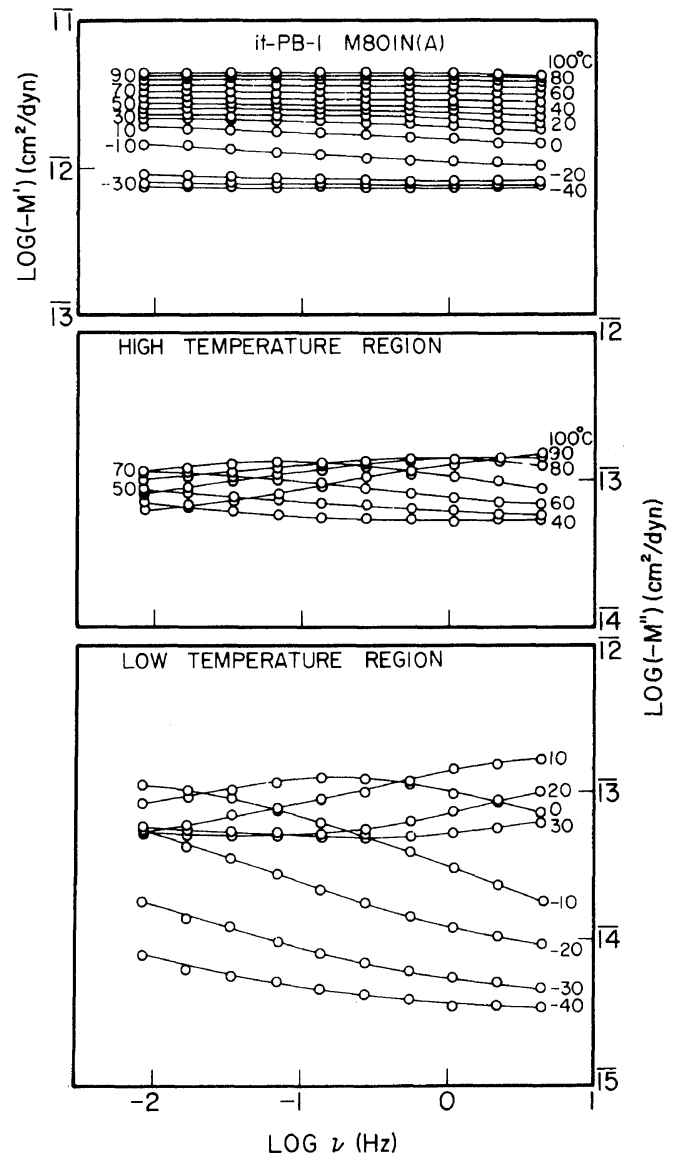

Figure 21. Temperature dependences of real and imaginary components of the complex dynamic stress-optical coefficient function of the it-PB-1 M801N(A) specimen.

suggest the good correspondence of the $\beta$ and $\alpha$ optical dispersions to the $\beta$ and $\alpha$ mechanical dispersions. The temperature depencence of $\log q_{\mathrm{T}}$ shown in Figures 24 and 25 is as weak as that of $\log b_{\mathrm{T}}$. However, this cannot be explained simple, because $q_{\mathrm{T}}$ must be a complicated function of the temperature dependence of the intrinsic birefringence and mechanical compliance of the responding elements of the $\alpha$ and $\beta$ retardations and the degree of crystallinity.

Arrhenius plots of $\log o_{\mathrm{T}}$ for spherulitic high density polyethylene also showed two linear regions which yielded activation energies of 17.2 and 24.2 $\mathrm{kcal} \mathrm{mol}^{-1}{ }^{21}$ These values and the associated temperature regions suggest the occurrence of $\beta$ and $\alpha$ optical dispersions. It is noteworthy that these 


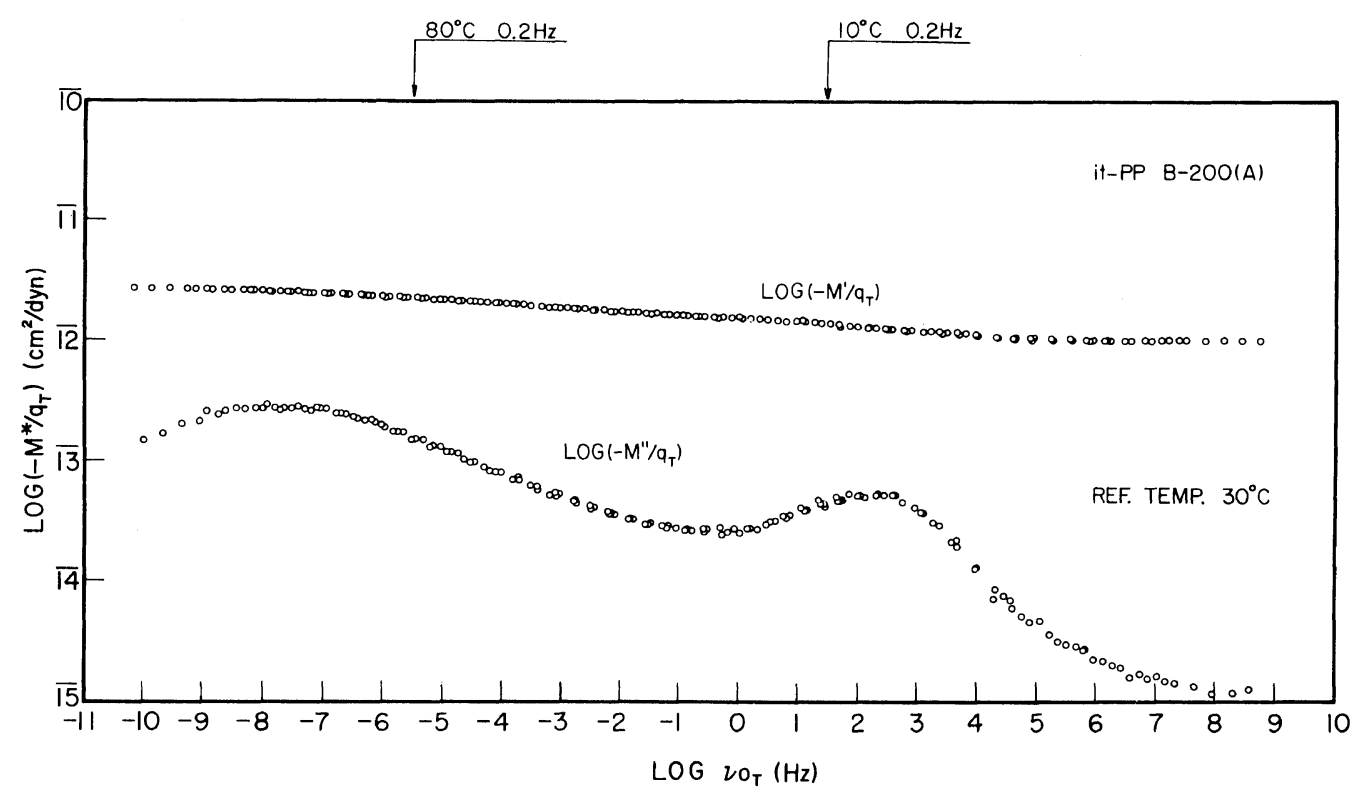

Figure 22. Superposed master curves of real and imaginary components of the complex dynamic stressoptical coefficient function of the it-PP B-200(A) specimen reduced to a reference temperature of $30^{\circ} \mathrm{C}$.

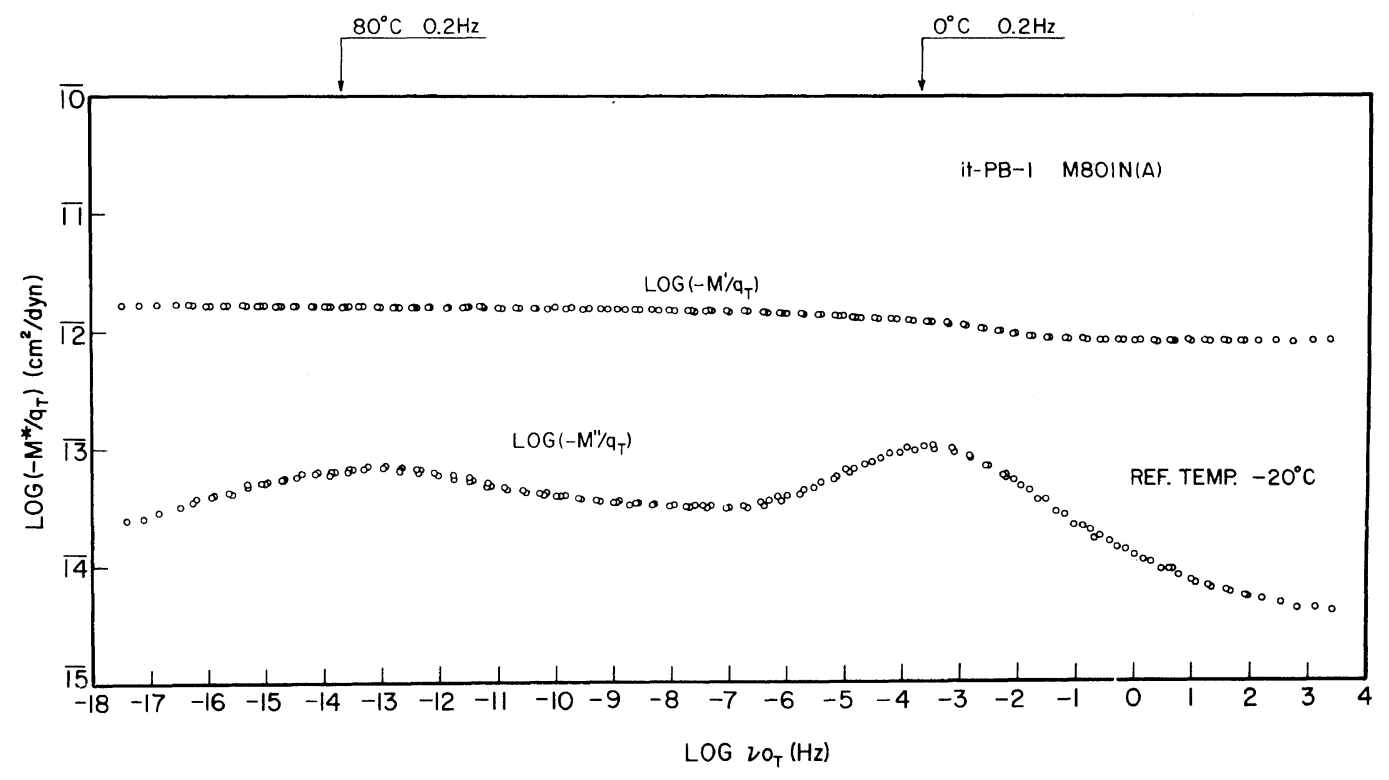

Figure 23. Superposed master curves of real and imaginary components of the complex dynamic stressoptical coefficient function of the it-PB-1 M801N(A) specimen reduced to a reference temperature of $-20^{\circ} \mathrm{C}$. 
The Mechanical Dispersion and the Dynamic Crystal Orientation of Spherulitic PP and PB-1

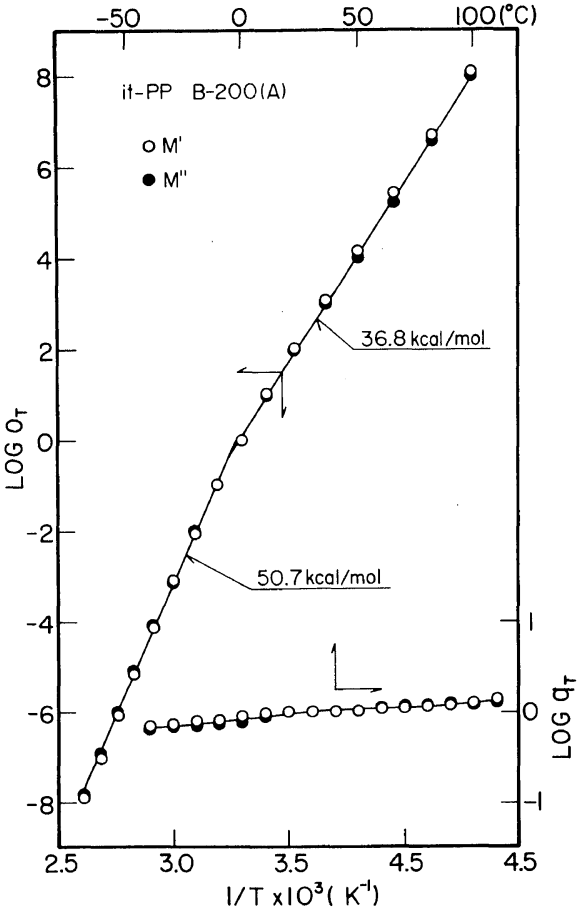

Figure 24. Arrhenius plots of the horizontal shift factor $\log o_{\mathrm{T}} v s$. reciprocal absolute temperature along with the temperature dependence of the vertical shift factor $\log q_{\mathrm{T}}$, both for composing the master curves in Figure 22 for the it-PP B-200(A) specimen.

activation energies are close to those of $\beta$ and $\alpha_{1}$ mechanical dispersions. Interestingly no optical dispersion corresponding to the $\alpha_{2}$ mechanical dispersion was observed even for polyethylene.

\section{Dynamic Crystal Orientation Dispersion}

In Figures 26 and 27 are shown the real components of the complex dynamic orientation distribution functions, $\Delta q_{j}^{\prime}\left(\zeta_{j}, 0\right)$, of the $j$-th crystal planes, determined as a function of the polar angle $\theta_{j}$ (open circles) by the dynamic X-ray diffraction technique $^{5}$ for the it-PP B-200(A) and it-PB-1 $\mathrm{M} 801 \mathrm{~N}(\mathrm{~A})$ specimens, respectively, as a frequency of $0.2 \mathrm{~Hz}$ and two different temperatures. The solid lines in the figures were drawn from $\Delta A_{l 0 n}^{\prime}$. That is, determining at first $\Delta \alpha_{l 0}^{j^{\prime}}$ from the observed $\Delta q_{j}{ }^{\prime}\left(\zeta_{j}, 0\right)$ by eq 18 , determining $\Delta A_{l 0 n}^{\prime}$ from $\Delta \alpha_{l 0}^{j^{\prime}}$ by eq 24 with $j=4$ for polypropylene and $j=2$ for polybutene-1, and then determining, $\Delta \alpha_{l 0}^{j^{\prime}}$ and further $\Delta q_{j}{ }^{\prime}\left(\zeta_{j}, 0\right)$ from $\Delta A_{l 0 n}^{\prime}$ by eq 24 and a dynamic

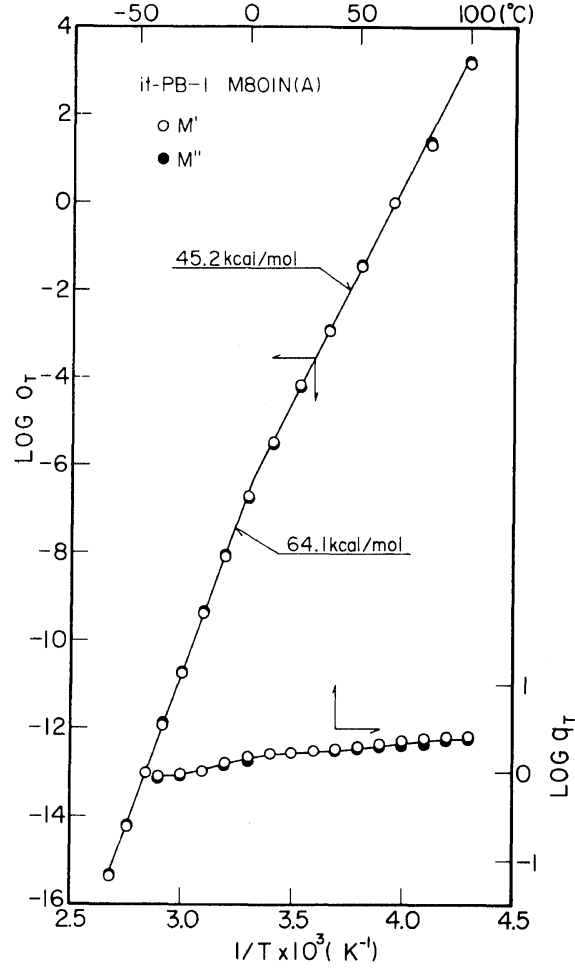

Figure 25. Arrhenius plots of the horizontal shift factor $\log o_{\mathrm{T}} v s$. reciprocal absolute temperature along with the temperature dependence of the vertical shift factor $\log q_{\mathrm{T}}$, both for composing the master curves in Figure 23 for the it-PB-1 M801N(A) specimen.

equation modified from eq 4 with finite series up to the 6th order for these particular polymers. The figures, show fairly good agreement between the observed and calculated results for the respective crystal planes, indicating a high accuracy of $\Delta A_{l 0 n}^{\prime}$ thus determined even for the polybutene- 1 specimen for which only two crystal planes, (110) and (300) crystal planes, are chosen with $j=2$.

Figures 28 and 29 show the temperature dependence of the real and imaginary components of the complex dynamic strain-orientation coefficient functions of the $j$-th crystal planes, $C_{j}{ }^{*}$ $\left(=C_{j}{ }^{\prime}+i C_{j}{ }^{\prime \prime}\right)$ defined by eq 21 , for the it-PP B200(A) and it-PB-1 M801N(A) specimens, respectively, both at a frequency of $0.2 \mathrm{~Hz}$. The dynamic measurements were performed only for the (110) and (040) crystal planes for the polypropylene specimen and the (110) and (300) crystal planes for the polybutene-1 specimen. The results for the crystal $c$ - 


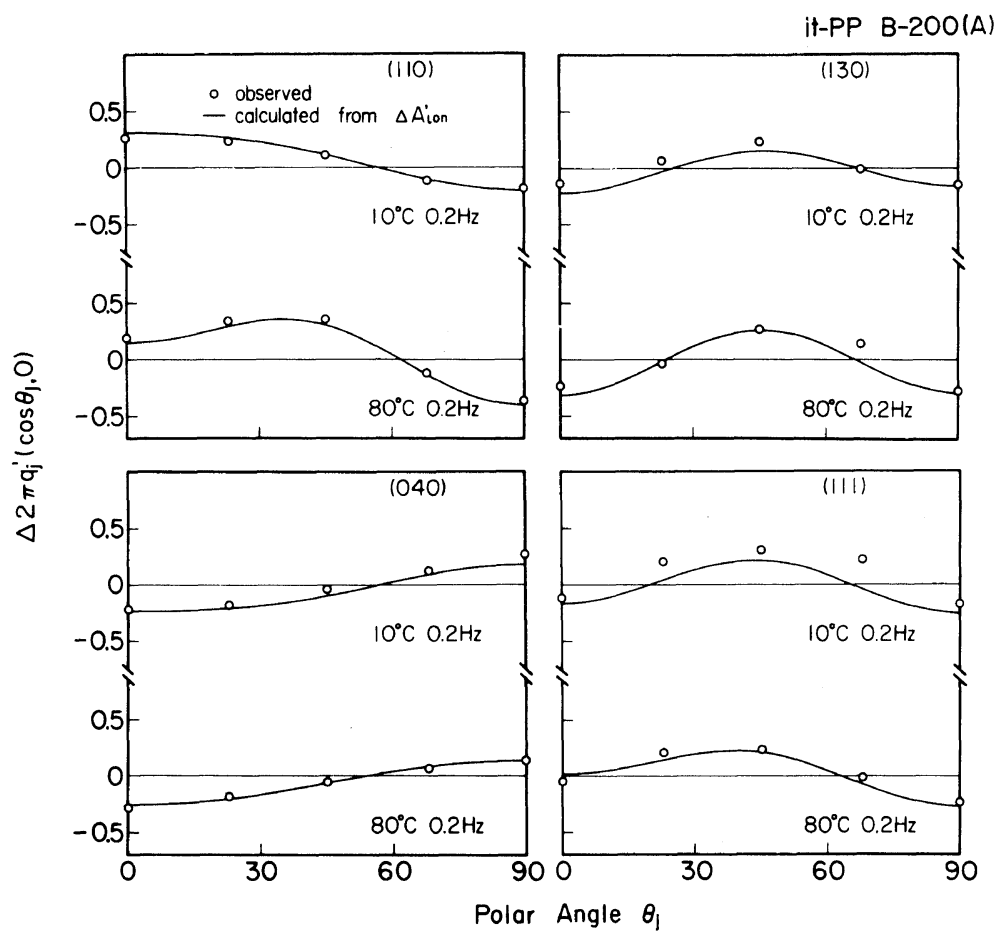

Figure 26. Comparison of the real component of the complex dynamic orientation distribution function $\Delta q_{j}^{\prime}\left(\zeta_{j}, 0\right)$ of the $j$-th crystal plane with those calculated from $\Delta A_{l 0 n}^{\prime}$ for the (110), (040), (130), and (111) crystal planes for the it-PP B-200(A) specimen at two different temperatures of 10 and $80^{\circ} \mathrm{C}$ at a given frequency of $0.2 \mathrm{~Hz}$.

it-PB-I M8OIN(A)
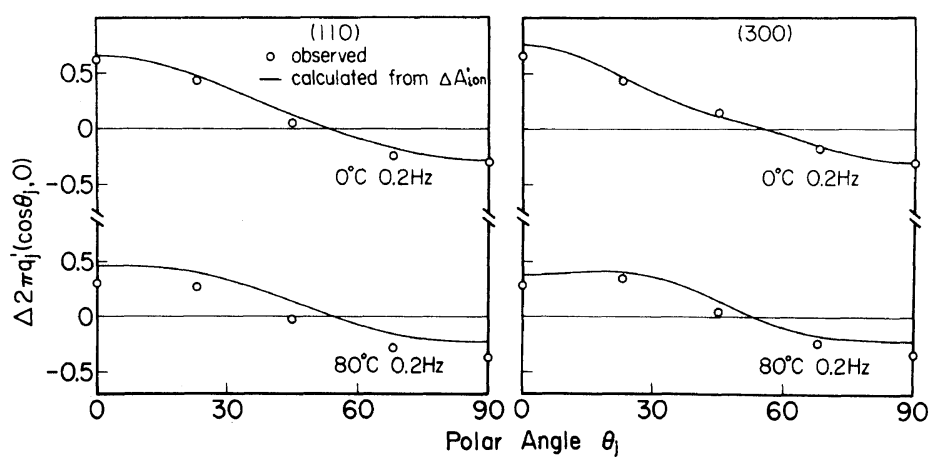

Figure 27. Comparison of the real component of the complex dynamic orientation distribution function $\Delta q_{j}{ }^{\prime}\left(\zeta_{j}, 0\right)$ of the $j$-th crystal plane with those calculated from $\Delta A_{l 0 n}^{\prime}$ for the (110) and (300) crystal planes for the it-PB-1 M801N(A) specimen at two different temperatures of 0 and $80^{\circ} \mathrm{C}$ at a given frequency of $0.2 \mathrm{~Hz}$.

axis for both specimens and the crystal $a^{*}$-axis for the polypropylene specimen were calculated from the observed data, using a relationship between the uniaxial 2nd-order orientation factors $F_{20}^{j}$ proposed by Wilchinsky ${ }^{16}$ as one of the simplest cases of eq 7 . As can be recognized from the figures, the strain- 
The Mechanical Dispersion and the Dynamic Crystal Orientation of Spherulitic PP and PB-1

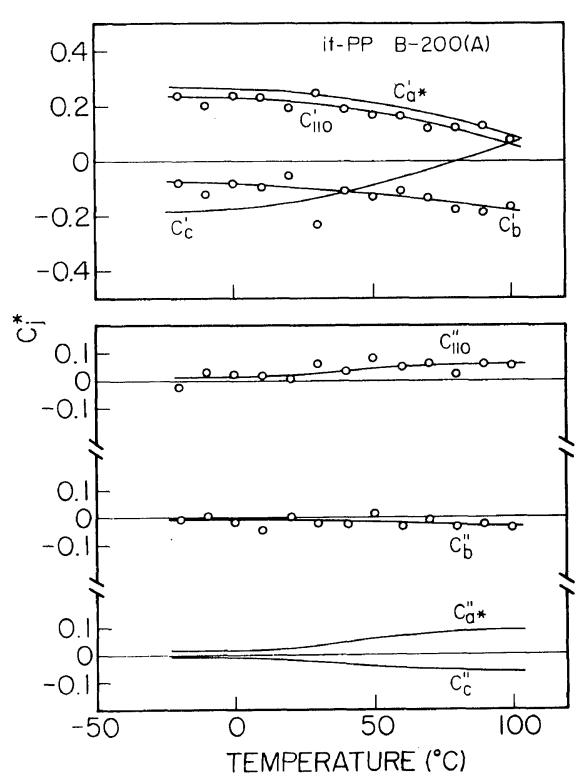

Figure 28. Temperature dependences of the complex dynamic strain-orientation coefficient function $C_{j}{ }^{*}$ for the $j$-th crystallographic axis of polypropylene crystal (monoclinic) for the it-PP B-200(A) specimen at a given frequency of $0.2 \mathrm{~Hz}$.

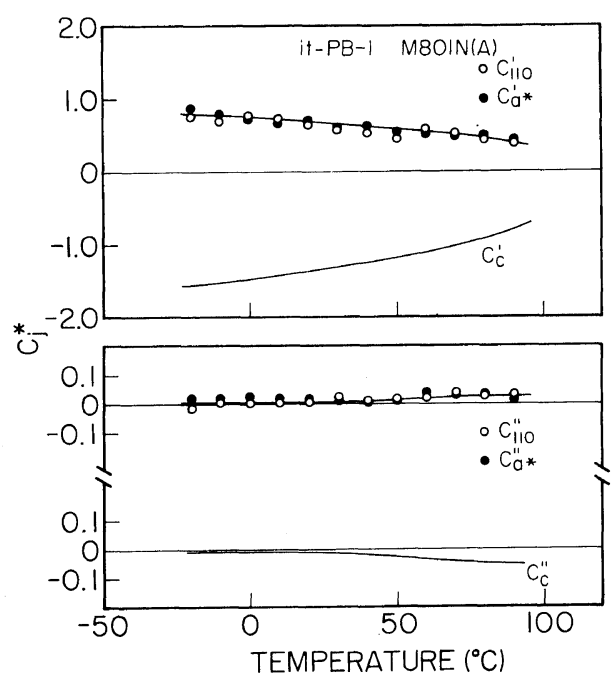

Figure 29. Temperature dependences of the complex dynamic strain-orientation coefficient function $C_{j}{ }^{*}$ for the $j$-th crystallographic axis of polybutene- 1 crystal (hexagonal) for the it-PB-1 M801N(A) specimen at a given frequency of $0.2 \mathrm{~Hz}$. orientation coefficient functions of the principal crystallographic axes definitely exhibit the orientation dispersion for both specimens over a temperature range covering the mechanical and optical dispersions in Figures 16 and 17 and Figures 22 and 23. This assumes that the mechanical and optical dispersions are related to the crystal orientation dispersions. The orientation dispersions, which are clearer at high temperature, have the feature that $C_{\mathrm{a}^{*}}^{*}$ remains positive, while $C_{\mathrm{c}}{ }^{*}$ remains mostly negative with the anomaly that the sign of $C_{\mathrm{c}}{ }^{\prime}$ changes from negative to positive for the polypropylene specimen at around $80^{\circ} \mathrm{C}$. These dynamic orientation characteristics of the principal crystallographic axes may be explained, as discussed later in detail in terms of a preferential orientation of lamellar axes ( $a^{*}$-axes) at low temperature, superposed with some reorientation mechanisms of crystal grains within the orienting lamellae to orient their crystal $c$-axes toward the stretching direction at high temperature.

Figures 30 and 31 show the real components of the complex dynamic orientation distribution functions of the crystal grains, $\Delta w^{\prime}(\xi, 0, \eta)$, determined from $A_{l 0 n}^{\prime}$ by eq 28 at a frequency of $0.2 \mathrm{~Hz}$ and at two different temperatures of 10 and $80^{\circ} \mathrm{C}$ for the itPP B-200(A) specimen and 0 and $80^{\circ} \mathrm{C}$ for the it-PB1 M801N(A) specimen, respectively. The static components, $w^{0}(\xi, 0, \eta)$ at $\lambda^{0}=1.05$, are also illustrated in the figures. The contour lines in $\Delta w^{\prime}(\xi, 0, \eta)$ show the intensities multiplied by a factor of $10^{3}$ relative to those for $w^{0}(\xi, 0, \eta)$, and the positive or negative sign of the contour lines corresponds to the dynamic increment or decrement in the orientation distribution function relative to the static component, $w^{0}(\xi, 0, \eta)$.

By comparing $\Delta w^{\prime}(\xi, 0, \eta)$ with $w^{0}(\xi, 0, \eta)$ in Figure 30 for the polypropylene specimen with the five types of preferential orientations of the crystal grains in Figure 11, it may be pointed out that type A orientation is associated with the loss of type $\mathrm{Cc}$ orientation at $10^{\circ} \mathrm{C}$ and that type $\mathrm{A}$ orientation is replaced by type $\mathrm{Ca}$ orientation also associated with the loss of type $\mathrm{Cc}$ orientation to a much greater extent at $80^{\circ} \mathrm{C}$ that at $10^{\circ} \mathrm{C}$. Thus, the dynamic lamellar orientation at $10^{\circ} \mathrm{C}$ must be accompanied with the dynamic lamellar tilting, probably at the polar zone of uniaxially deformed spherulites, in counter-balance of a large loss of the dynamic orientation of the crystal grains in the type $\mathrm{Cc}$ 


\section{it-PP B-200 (A)}

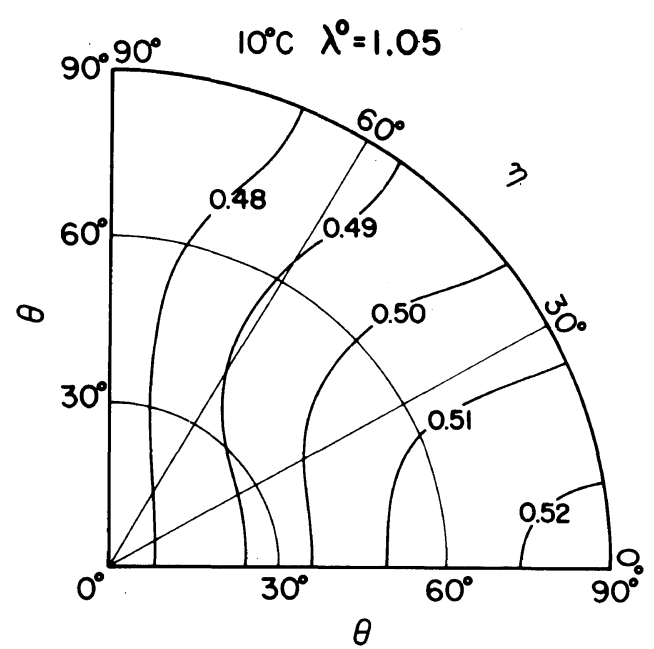

(a)

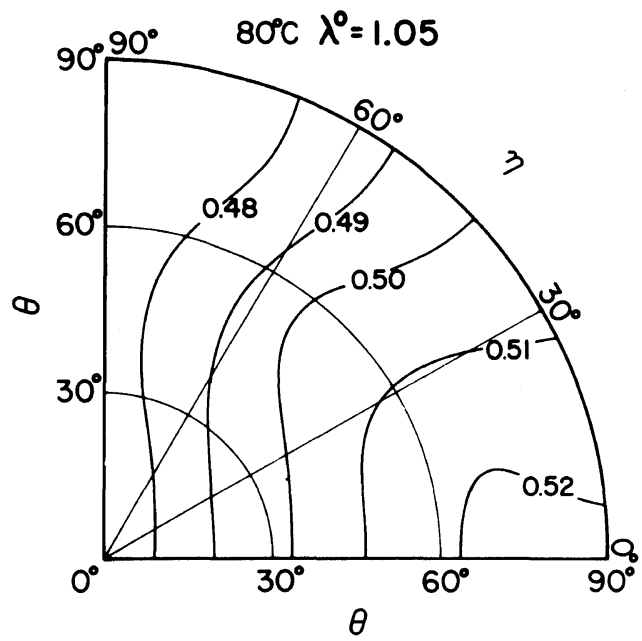

(c)

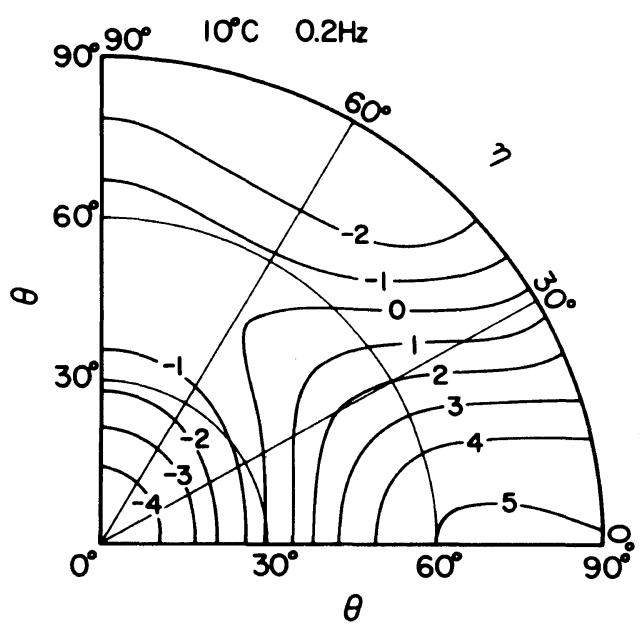

(b)

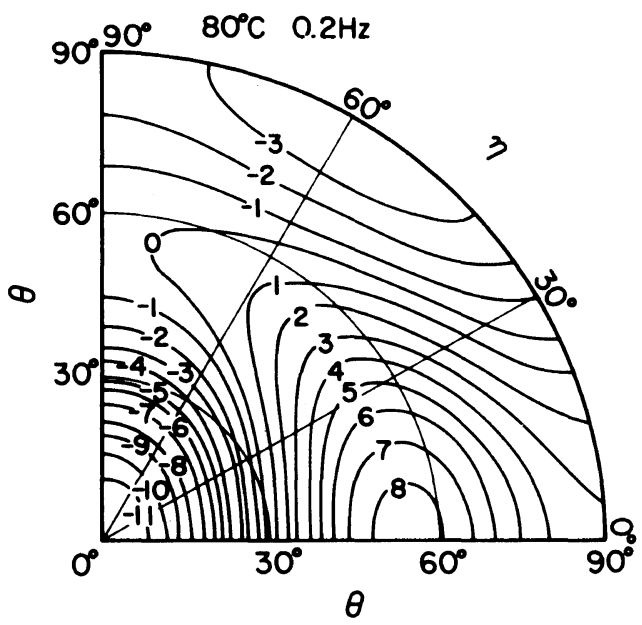

(d)

Figure 30. Real component of the complex dynamic orientation distribution function $\Delta w^{\prime}(\xi, 0, \eta)$ of the crystal grains determined from $\Delta q_{j}{ }^{\prime}\left(\zeta_{j}, 0\right)$ in Figure 26 and further from $\Delta A_{l 0 n}^{\prime}$ for the it-PP B-200(A) specimen at two different temperatures of 10 and $80^{\circ} \mathrm{C}$ at a given frequency of $0.2 \mathrm{~Hz}$. Corresponding static components $w^{0}(\xi, 0, \eta)$ at the two temperatures are also shown. 


\section{it-PB-1 M80IN (A)}

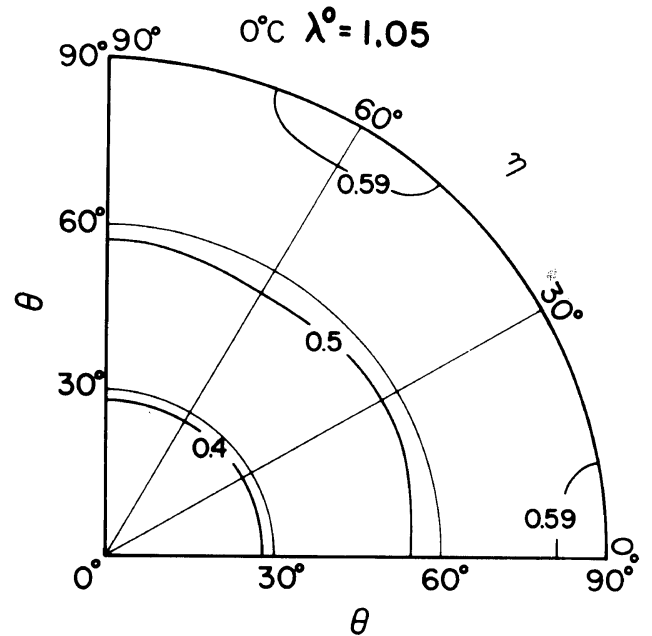

(a)

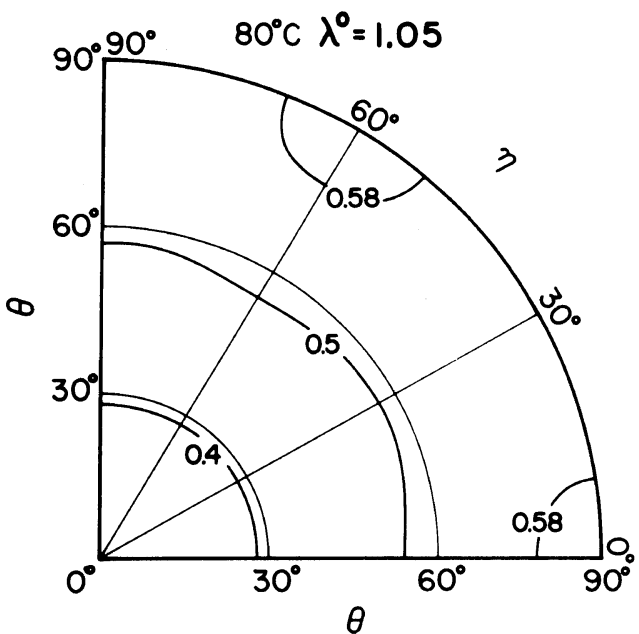

(c)

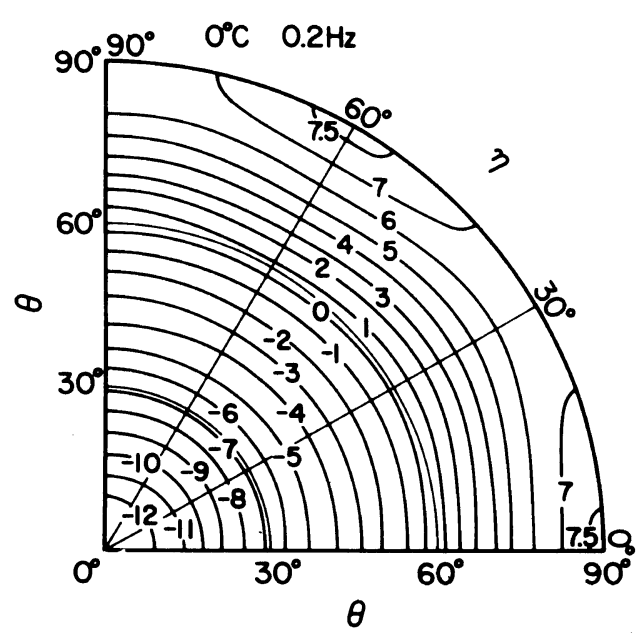

(b)

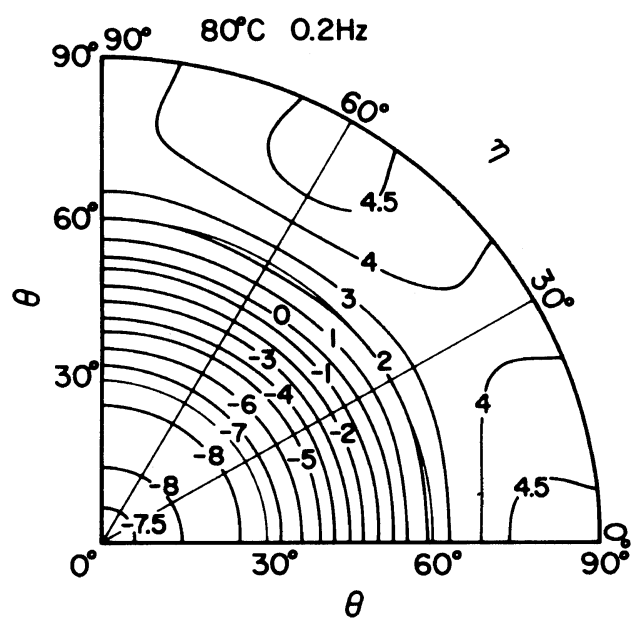

(d)

Figure 31. Real component of the complex dynamic orientation distribution function $\Delta w^{\prime}(\xi, 0, \eta)$ of the crystal grains determined from $\Delta q_{j}{ }^{\prime}\left(\zeta_{j}, 0\right)$ in Figure 27 and further from $\Delta A_{i 0 n}^{\prime}$ for the it-PB-1 M801N(A) specimen at two different temperatures of 0 and $80^{\circ} \mathrm{C}$ at a given frequency of $0.2 \mathrm{~Hz}$. Corresponding static component $w^{0}(\xi, 0, \eta)$ at the two temperatures are also shown. 


\section{K. Fujita et al.}

orientation. By comparing $\Delta w^{\prime}(\xi, 0, \eta)$ with $w^{0}(\xi, 0, \eta)$ in Figure 31 for the it-PB-1 M801N(A) specimen, a similar explanation may be made putting somewhat less emphasis on the lamellar tilting mechanism for the polybutene-1 specimen than for the polypropylene specimen.

Phenomenologically, the negative sign of the complex dynamic stress-optical coefficient function, $M^{*}\left(=M^{\prime}+i M^{\prime \prime}\right)$ for the polypropylene and polybutene- 1 specimens, in contrast to the positive sign for the polyethylene specimen at relatively high temperature, can be understood from the $\Delta n v s . \lambda$ curves in Figures 5 and 6 in terms of their negative derivatives at $\lambda=\lambda^{0}=1.033$ for both specimens. However, the $\beta$ optical dispersion with negative $M^{*}$ at relatively low temperature may be interpreted in terms of the dynamic orientation dispersion of the crystal lamellae having negative intrinsic birefringence, as definitely confirmed in Figures 30(b) and $31(\mathrm{~b})$ in terms of $\Delta w^{\prime}(\xi, 0, \eta)$ and the corresponding reduced frequencies, as indicated by the right-hand side arrows in Figures 22 and 23. The $\alpha$ optical dispersion with positive $M^{*}$ for the spherulitic high-density polyethylene at relatively high temperature may be interpreted in terms of dynamic orientation dispersions of the crystal grains within the orienting lamellae, i.e., type $\mathrm{Ca}$ and $\mathrm{Cb}$ orientations of the crystal grains, as demonstrated in the previous paper ${ }^{3}$ in terms of $\Delta w^{\prime}(\xi, 0, \eta)$, exhibited positive increments in the dynamic birefringence. In contrast, the $\alpha$ optical dispersion with negative $M^{*}$ for the polypropylene and polybutene-1 cannot be explained simply, unless the dynamic decrement of the type $\mathrm{Cc}$ orientation of the crystal grains dominates the dynamic increment of the type $\mathrm{Cb}$ orientation of the crystal grains, as suggested in Figures 30(d) and 31(d) in terms of $\Delta w^{\prime}(\xi, 0, \eta)$ and their associated reduced frequencies indicated by the left-hand side arrows in Figures 22 and 23.

Figure 32 is a schematic diagram explaining the $\alpha$ and $\beta$ optical dispersions, both having negative sign for the polypropylene and polybutene-1 specimens. Here 'positive' and 'negative' correspond, respectively, to the dynamic increment of type $\mathrm{Cb}$ orientation yielding positive sign and the dynamic decrement of type $\mathrm{Cc}$ orientation giving negative sign in the dynamic birefringence dispersion. The structural origin of the dynamic decrement of the type $\mathrm{Cc}$ orientation in terms of the dynamic deformation of the spherulitic texture is uncertain, at present.
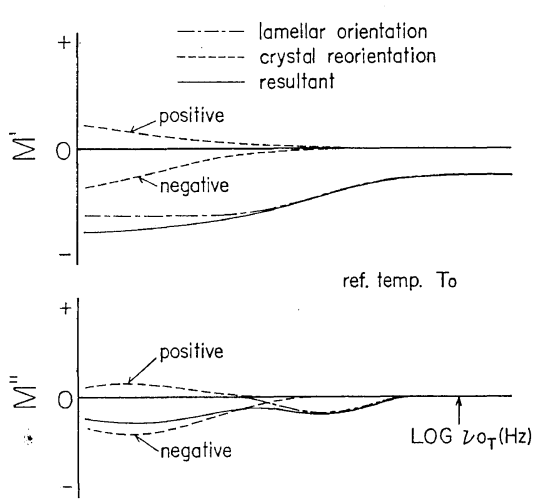

Figure 32. Schematic diagram assigning the $\beta$ and $\alpha$ optical dispersions in the complex dynamic stress-optical coefficient function $M^{*}$ both with a negative sign of the function for the polypropylene and polybutene-1 specimens.

Figures 33 and 34 show the crystalline and noncrystalline contributions to the master curves of the complex dynamic strain-optical coefficient function, $K^{*}=\partial \Delta n^{*} /\left.\partial \varepsilon^{*}\right|_{\lambda=\lambda^{0}}$, which were calculated from $J^{*}$ and $M^{*}$ in Figures $16,17,22$, and 23 with the relation $K^{*}=M^{*} / J^{*}$. The crystalline and noncrystalline contributions to $K^{*}$ were calculated assuming the additivity in volume; i.e.,

$$
\begin{aligned}
K_{T}^{*}(i \omega)= & X_{\mathrm{c}}(T) K_{\mathrm{c}, T}^{*}(i \omega)+\left[1-X_{\mathrm{c}}(T)\right] K_{\mathrm{am}, T}^{*}(i \omega) \\
= & X_{\mathrm{c}}(T) \sum_{k}^{a^{*}, b}\left(n_{k}-n_{\mathrm{c}}\right)_{T} C_{k, T}^{*}(i \omega) \\
& +\left[1-X_{\mathrm{c}}(T)\right] \Delta_{\mathrm{am}, T}^{0} C_{\mathrm{am}, T}^{*}(i \omega)
\end{aligned}
$$

where $K_{\mathrm{c}, T}^{*}$ and $K_{\mathrm{am}, T}^{*}$ are the crystalline and noncrystalline contributions to $K_{T}{ }^{*}$ and $X_{\mathrm{c}}(T)$ is the crystalline volume fraction at a given temperature T. $C_{k, T}^{*}$ and $C_{\mathrm{am}, T}^{*}$ are the complex dynamic strainorientation coefficient functions for the $k$-th crystallographic axis and the noncrystalline chain segment, respectively, and $\left(n_{k}-n_{\mathrm{c}}\right)$ and $\Delta_{\mathrm{am}, T}^{0}$ are crystalline and noncrystalline intrinsic birefringence again all the given temperature $T$.

For the numerical calculation of the crystalline contribution $K_{\mathrm{c}, T}^{*}(i \omega)$, the intrinsic birefringence, $\left(n_{\mathrm{a}}^{*}-n_{\mathrm{c}}\right)=-0.0352$ and $\left(n_{\mathrm{b}}-n_{\mathrm{c}}\right)=-0.0349$ proposed by Takahara et al. $^{31}$ for the monoclinic polypropylene crystal and $\left(n_{\mathrm{c}}-n_{\mathrm{a}}{ }^{*}\right)=\left(n_{\mathrm{c}}-n_{\mathrm{b}}\right)=$ 0.0146 calculated for the hexagonal polybutene- 1 crystal from the bond-polarizabilities by BunnDaubeny ${ }^{32}$ and Vulk $^{33}$ and from the hexagonal 
The Mechanical Dispersion and the Dynamic Crystal Orientation of Spherulitic PP and PB-1

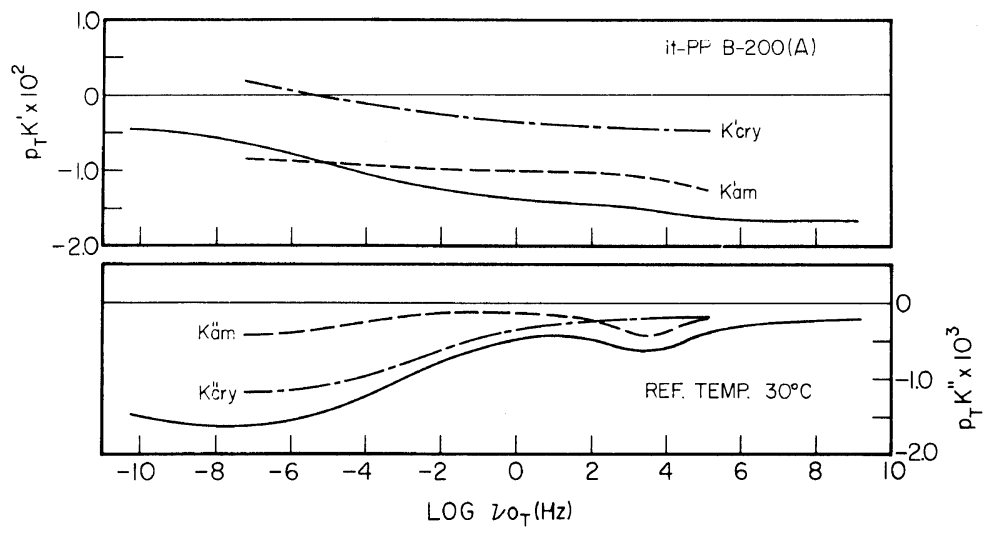

Figure 33. Crystalline and noncrystalline contributions to the master curves of the complex dynamic strain-optical coefficient function $K^{*}$, calculated from the results of $J^{*}, M^{*}$, and $C_{j}^{*}$ in Figures 16, 22, and 28 for the it-PP B-200(A) specimen.

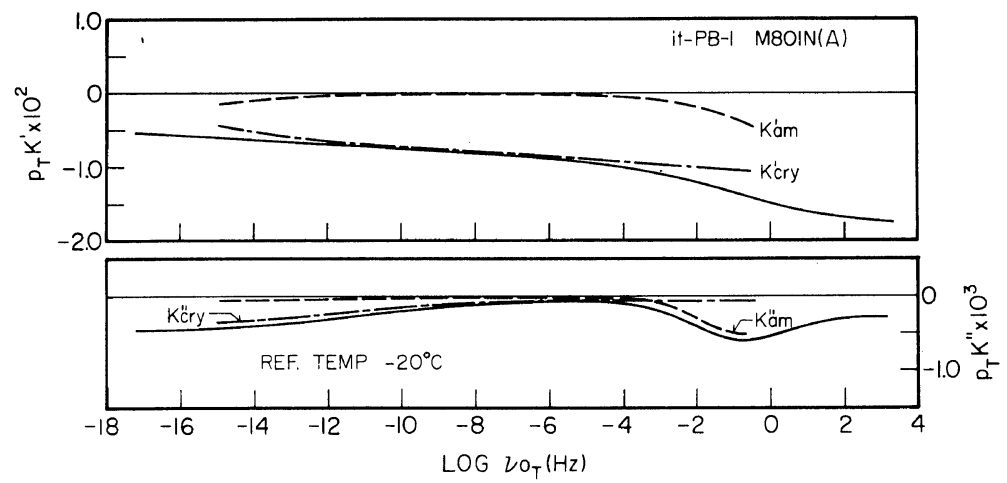

Figure 34. Crystalline and noncrystalline contributions to the master curves of the complex dynamic strain-optical coefficient function $K^{*}$, calculated from the results of $J^{*}, M^{*}$, and $C_{j}{ }^{*}$ in Figures 17, 23, and 29 for the it-PB-1 M801N(A) specimen.

crytal structure by $\mathrm{Natta}^{34}$ were used, assuming them to be temperature independent. The intrinsic birefringence of 0.0146 for the hexagonal polybutene- 1 crystal is considerably smaller than those proposed by Oda et al..$^{35}(0.0241)$ and by Onogi et $a l^{29}(0.034)$. The temperature dependence of $X_{\mathrm{c}}(T)$ was determined from that of the static component, $q_{j, T}^{0}\left(\zeta_{j}, 0\right)$, observed simultaneously with $\Delta q_{j, T}^{*}\left(\zeta_{j}, 0\right)$ for the determination of $C_{j, T}^{*}\left(i \omega_{0}\right)$ at a frequency of $0.2 \mathrm{~Hz}$ in Figures 28 and 29. $C_{j, T}^{*}\left(i \omega_{0}\right)$ thus determined was reduced to $C_{j, T_{0}}^{*}\left(i \omega c_{T}\right)$ at a reference temperature $T_{0}$, assuming that $c_{\mathrm{T}}=o_{\mathrm{T}}$.

As can be seen from Figures 33 and 34, in contrast to $M^{*}$ in Figures 22 and 23 , a rapid decrease of $K_{\mathrm{c}}{ }^{\prime}$ in negative sign in the vicinity of the $\alpha$ optical dispersion region, even displaying a change in sign from negative to positive for the itPP B-200(A) specimen, must be noticed. This rapid decrease of $K_{\mathrm{c}}{ }^{\prime}$ can be understood as a consequence of the activation of the type $\mathrm{Cb}$ orientation, though the negative sign of $K_{\mathrm{am}}^{*}$ over the whole range of reduced frequency gives rise an additional problem difficult to explain.

The dynamic rheo-optical properties investigated above may be summarized as follows: Two mechanical dispersions were found with good correspondence to two optical dispersions, designated as $\alpha$ and $\beta$ dispersions at low frequencies and high temperatures and at high frequencies and low temperatures, respectively, for the spherulitic polypro- 
pylene and polybutene- 1 specimens. In contrast, three mechanical dispersions, $\alpha_{1}, \alpha_{2}$, and $\beta$ mechanical dispersions were detected for the spherulitic high-density polyethylene with good correspondence to two optical dispersions; i.e. no optical dispersion corresponds to the $\alpha_{2}$ mechanical dispersion was observed.

The complex dynamic stress-optical coefficient function, $M^{*}\left(=M^{\prime}-i M^{\prime \prime}\right)$ was observed as negative in its sign for every poly-alpha-olefin specimen in the $\beta$ optical dispersion region, but as positive and negative in its sign for the polyethylene and the other poly-alpha-olefin specimens, respectively, in the $\alpha$ dispersion region. In order to assign the $\alpha$ and $\beta$ mechanical and optical dispersions of the poly-alpha-olefins, the dynamic complex orientation distribution function of crystal grains, $\Delta w^{*}(\xi, 0, \eta)$, was observed as a funtion of the Euler angles, $\phi, \theta$, and $\eta$, at particular frequencies and temperatures covering the dispersion regions. The following assignments were made: The $\beta$ mechanical and optical dispersions can be assigned to the dynamic orientation dispersion of crystal lamellae constituting the spherulitic crystalline texture with negative intrinsic birefringence, i.e. inter-lamellar crystal-grain-boundary phenomena possibly associated with orientational and/or distorsional dispersions of noncrystalline material between the lamellae. The $\alpha$ mechanical and optical dispersions can be assigned to the dynamic reorientation dispersion of crystal grains within the orienting lamellae in such preferential manners of $c$-axis orientation as designated by type $\mathrm{Ca}, \mathrm{Cb}$, and $\mathrm{Cc}$. For the polyethylene specimen, the dynamic increment in type $\mathrm{Ca}$ and $\mathrm{Cb}$ orientations result in the positive sign in $M^{*}$. For the polypropylene and polybutene-1 specimens, however, the dynamic decrement of type $\mathrm{Cc}$ orientation must predominate over the dynamic increment of the type $\mathrm{Cb}$ orientation to result in the negative sign in $M^{*}$. The more obvious is the $\alpha$ dispersion, but the less obvious is the $\beta$ dispersion, both as the specimen changes from polybutene-1 to polypropylene and polyethylene.

Acknowledgements. This series of rheo-optical studies on the deformation mechanisms of semicrystalline polymers has been supported in part by a grant from the U.S.-Japan Cooperative Research Program of the National Science Foundation and the Japan Society for the Promotion of Science.
The authors are also indebted to the Nippon Gosei Kagaku Co., Ltd., Osaka, Japan and the Dai-cell Chemical Industries Ltd., Osaka, Japan, for financial support through a scientific grant.

\section{REFERENCES}

1. K. Fujita, S. Suehiro, S. Nomura, and H. Kawai, Polym. J., 14, 545 (1982).

2. K. Shiro, K. Fujita, S. Suehiro, and H. Kawai, Polymer (London), in press.

3. K. Fujita, H. Niwa, S. Nomura, and H. Kawai, $J$. Polym. Sci., Polym. Phys. Ed., in press.

4. T. Kyu, N. Yasuda, 'M. Tabushi, S. Nomura, and H. Kawai, Polym. J., 8, 565 (1976).

5. S. Suehiro, T. Yamada, H. Inagaki, and H. Kawai, Polym. J., 10, 315 (1978).

6. R. S. Stein and M. B. Rhodes, J. Appl. Phys., 31, 1873 (1960).

7. R. J. Samuels, J. Polym. Sci., A, 3, 1741 (1965).

8. R. J. Samuels, J. Polym. Sci., C, 20, 253 (1967).

9. S. L. Aggarwal, Physical Constants of Poly-alphaolefins in "Polymer Handbook," J. Brandrup and E. H. Immergut, Ed., John Wiley \& Sons, New York, 1975.

10. S. Suehiro, T. Yamada, T. Kyu, K. Fujita, T. Hashimoto, and H. Kawai, Polym. Eng. Sci., 19, 929 (1979).

11. T. Ito, T. Oda, H. Kawai, T. Kawaguchi, D. A. Keedy, and R. S. Stein, Rev. Sci. Instr., 39, 1847 (1968).

12. R. J. Roe and W. R. Krigbaum, J. Chem. Phys., 40, 2608 (1964).

13. W. R. Krigbaum and R. J. Roe, J. Chem. Phys., 41, 737 (1964).

14. R. J. Roe, J. Appl. Phys., 36, 2024 (1965).

15. R. A. Sack, J. Polym. Sci., 54, 543 (1961).

16. Z. W. Wilchinsky, J. Appl. Phys., 31, 1969 (1960).

17. H. Kawai, Rheol. Acta, 14, 27 (1975).

18. K. Sasaguri, M. B. Rhodes, and R. S. Stein, J. Polym. Sci., B, 1, 571 (1963).

19. K. Sasaguri, S. Hoshino, and R. S. Stein, J. Appl. Phys., 35, 47 (1964).

20. K. Sasaguri, R. Yamada, and R. S. Stein, J. Appl. Phys., 35, 3188 (1964).

21. T. Kyu, M. Yamada, S. Suehiro, and H. Kawai, Polym. J., 12, 809 (1980).

22. J. A. Faucher, Trans. Soc. Rheol., 3, 81 (1959).

23. K. Nagamatsu, Kolloid Z., 172, 141 (1960).

24. Y. Wada, J. Phys. Soc., Jpn., 16, 1226 (1961).

25. Y. Ishida, Y. Ueno, S. Togami, and M. Matsui, Kolloid Z., 199, 70 (1964).

26. T. Hara and K. Okano, J. Phys. Soc., Jpn., 20, 1291 (1965).

27. S. Onogi, Y. Fukui, T. Asada, and Y. Naganuma, Proceedings of the 5th International Congress on 
The Mechanical Dispersion and the Dynamic Crystal Orientation of Spherulitic PP and PB-1

Rheology, 4, 87 (1970).

28. T. Asada, J. Sasada, and S. Onogi, Polym. J., 3, 350 (1972).

29. A. Tanaka, N. Sugimoto, T. Asada, and S. Onogi, Polym. J., 5, 529 (1975).

30. M. Pizzoli, N. G. McCrum, and F. C. Chen, 3rd Convention of Italian Science of Macromolecules, 1977, p 31.

31. H. Takahara, T. Yamada, and H. Kawai, J. Soc.
Fiber Sci. Tech., Jpn., 23, 571 (1967).

32. C. W. Bunn and R. de Daubeny, Trans. Faraday Soc., 50, 1173 (1954).

33. M. F. Vulk, Opt. Spectrosk., 2, 494 (1957).

34. G. Natta, P. Corradini, and I. W. Bassi, Nuovo Cimento, Suppl., 15, 52 (1960).

35. T. Oda, M. Maeda, S. Hibi, and S. Watanabe, Kobunshi Ronbunshu, 32, 291 (1974). 